(C) 2000 International Press

Adv. Theor. Math. Phys. 4 (2000) 823-891

\title{
Cohomological Field Theories with Kähler Structure
}

\author{
Jae-Suk Park \\ Institute for Theoretical Physics \\ University of Amsterdam \\ Valckenierstraat 65, 1018 XE Amsterdam \\ and \\ Department of Physics, Columbia University \\ 538 West 120th Street, New York, N.Y. 10027 \\ jspark@phys.columbia.edu
}

\begin{abstract}
This paper is devoted to a general and self-contained approach to any cohomological field theory with Kähler structure.
\end{abstract}

e-print archive: http://xxx.lanl.gov/ 


\section{Introduction}

Historically the cohomological field theory first has been introduced as a twisted version of global space-time supersymmetric quantum field theory, specifically the $N=2$ supersymmetric Yang-Mills theory in four dimensional space-time [1]. The global space-time supersymmetry, by definition, requires the existence of a spinor which is constant everywhere on the space-time manifold $M$. A spinor does exist on a spin manifold. A spin manifold, however, rarely admits a constant spinor. The canonical way overcoming the above difficulty is localizing the supersymmetry, which procedure almost magically introduces (super-)gravity into the picture.

There is a second option called twisting, meaning that one defines a new Lorentz symmetry group by a suitable combination of the original Lorentz symmetry with an internal global symmetry of the theory. As a result, the supercharges transform differently under the new Lorentz symmetry, which typically includes some components which transform as scalars. Such a scalar component $Q$, which is nilpotent $Q^{2}=0$, is regarded as a supercharge of the twisted theory. The resulting theory is well-defined on an arbitrary space-time since there are no global obstruction for a scalar and enjoys general covariance without gravity. The path integral of the theory depends only on the global cohomology of $Q$, provided that one uses $Q$-invariant observables, which property coined the adjective cohomological [2].

A twisted theory is closely related to the underlying space-time supersymmetric theory. Namely the path integral of the twisted theory computes a certain chiral (or BPS) sector of physical amplitudes [3], [4]. This is due to the trivial holonomy of flat space-time where the physical theory is usually defined. Then twisting is a physically invisible operation. The typical physical application of a twisted theory is a non-perturbative test of certain duality utilizing the semi-classical exactness of the path integral. Two famous examples are given by mirror symmetry [5], [6] and the $S$-duality of $N=4$ supersymmetric Yang-Mills theory in four-dimensions [7]. The twisted version of four dimensional $N=2$ supersymmetric Yang-Mills theory - the DonaldsonWitten theory [1], [8], also provided crucial hints [9], [10] on the cele- 
brated Seiberg-Witten solutions of the original $N=2$ theory [11]. ${ }^{1}$

In general we may forget about the underlying physical origin of a cohomological field theory and define the theory as a quantum field theory with global fermionic symmetry. Such a theory may not be directly obtainable as a twisted version of underlying space-time supersymmetric theory. The most fundamental property of a quantum field theory with global fermionic symmetry is the fixed point theorem of Witten [3], [13]. Almost all the other properties of cohomological field theory can be obtained as certain a lemma of the theorem.

In this paper we develop a general approach which identifies any cohomological field theory with $0+0$-dimensional supersymmetric sigma model. Being in zero-dimensions the (space-time) supersymmetry simply means global fermionic symmetry. The target space of our sigmamodel may be some function space $X$ in the theorem quoted above. Such a space may be any (non-linear or linear and finite or infinite dimensional) endowed with any of

\section{Riemannian $\supset$ Kähler $\supset$ hyper-Kähler}

structures. Actually the above structures may not be regarded as $a$ priori notions. The cohomological field theory can be classified by the number $N_{c}=\left(\mathcal{N}_{c}^{+}, N_{c}^{-}\right)$of global supercharges, where we have $N_{c}^{+}+N_{c}^{-}$ independent mutually nilpotent fermionic charges and $N_{c}^{ \pm}$denote the number of charges carrying fermionic (or ghost) numbers \pm 1 . Then we have the following sequence of fermionic symmetries

$$
N_{c}^{+}=1 \supset N_{c}^{+}=2 \supset N_{c}^{+}=4,
$$

which determine the sequence of geometrical structures (1.1). ${ }^{2}$

\footnotetext{
${ }^{1}$ We must stress here that solutions of the underlying physical theory provides us with invaluable insights in the mathematical problem defined by the twisted theory. Perhaps one of the most beautiful properties of quantum field theory is that the theory depends on a scale. The equally beautiful property of cohomological field theory is that the theory does not depends on a scale. Thus the mathematical problem defined by the latter theory can be solved in terms of the former theory in different scale where its relevant degrees of freedom is, often, completely different from the original microscopic ones. The historical example is, of course, the Donaldson versus Seiberg-Witten invariant [12].

${ }^{2}$ The above correspondence is originally due to supersymmetric sigma models in two-dimensions [14], [15], [16]. In certain respects, such a correspondence in zero-
} 
In this paper we specialize to models with a Kähler structure. Those models are quite general and allow us to have very compact formulations. The initial data will be some function space $X$ endowed with a complex structure compatible with the supersymmetry. Then most of the other structures of the models can be fixed. We will introduce three types of models, two with $N_{c}=(2,0)$ and one with $N_{c}=(2,2)$ symmetry, and establish general interrelations. For each type we will consider non-linear $X$ and linear or non-linear $X$ with a group $\mathcal{G}$ acting on $X$. In due course the relation between our construction and two-dimensional supersymmetric field theory will become obvious. This opens up possibilities of stringy generalizations of those differential-topological invariants defined by cohomological field theory. This chapter may be also viewed, after slight modifications, as an unorthodox introduction to two-dimensional supersymmetric field theories. Our presentation for models with a group action will parallel the original literature on $N_{w s}=(2,2)$ and $N_{w s}=(2,0)$ gauged linear sigma-models in two dimensions [18], [19]. We should also mention the influential paper of Witten on supersymmetry and Morse theory [20] dealing with $(0+1)$ dimensional supersymmetric sigma models, which can be regarded as the origin of cohomological field theory. ${ }^{3}$

Perhaps our definition for cohomological field theory as a zero dimensional sigma model might be confusing. If the target space $X$ is the function space of certain fields on a manifold $M$ we have a traditional cohomological field theory on $M$. As some general literature for cohomological field theory we refer to [2] and [21] for short but lucid introductions. We refer to [22] for a general reference for the Riemannian version of $\mathcal{N}_{c}=(2,0)$ models. For the Riemannian version of $\mathcal{N}_{c}=(2,2)$ model, called balanced cohomological field theory, we refer to [23]. For a mathematician the path integral of a cohomological field theory is Mathai-Quillen formalism of integral representation of Thom class [24], [21]. Though we will never refer to Mathai and Quillen, our (path) integral formula can be viewed as Kähler version of

dimensional models is more striking since we do not need any underlying geometrical objects like the two-dimensional space-time. Actually the sequence (1.2) leads to more general geometrical structures including torsion [17]. However, the author is not aware of any examples of traditional cohomological field theory with torsion in the space of fields.

${ }^{3}$ It is ironical since his construction can be regarded as, in our viewpoints, a generalized cohomological field theory. 
Mathai-Quillen formalism. More precisely our formua should be viewed as a certain equivariant generalization of Fulton and MacPerson's intersection theory [25]. For a physicist a cohomological field theory a supersymmetric gauged sigma model in $(0+0)$-dimensions. Though we will never use the superspace formalism our construction is equivalent to $N=2$ superspace formalism.

The most fundamental property of a quantum field theory with a global fermionic symmetry is the fixed point theorem of Witten. Almost all the other properties of cohomological field theory can be obtained as a certain lemma of the theorem. We refer to the original references [3], [13] for this crucial theorem.

\section{Standard Models of Cohomological Field Theory}

This and the next chapters are devoted to an elementary and selfcontained introduction to cohomological field theory. Though elementary, we will develop the most general construction of cohomological field theory involving Kähler geometry.

In this chapter we consider supersymmetric sigma models in $(0+0)$ dimensions, whose target space is a compact complex Kähler manifold $X$. Those models may be regarded as the quantum theory of single point-like "instanton" - the point-like event of $X$ or point-like instanton probes of the classical geometry of $X$ by means of the path integral. The space of all bosonic field will be the configuration space of the instanton, which is a copy of the manifold $X$. We will start from the simplest $N_{c}=(2,0)$ model as a toy model. A slightly more complicated $N_{c}=(2,2)$ model follows. Then we generalize it to another $N_{c}=(2,0)$ model. We will survey how those supersymmetric theories probe or give rise to the classical geometry of Kähler manifolds $X$, its tangent bundle $T X$ and holomorphic Hermitian vector bundle $\mathbb{E}$ over $X$. The models to be covered here will be used as the prototypes of all the other more elaborated models to be introduced later. We refer to the models in this chapter as standard models since any cohomological field theory will reproduce to one of those models if it is "generic". 
We follow a typical procedure of defining supersymmetric field theory, namely introducing bosonic fields, supercharges with their algebra, fermionic superpartners, supersymmetric action functional, and studying path integrals. Due to the triviality of the model everything can be made completely rigorous. Assuming existence of nil-potent supercharges, a simple application of Poincaré lemma leads to an appropriate supersymmetric action functional. All the other geometrical structures then naturally follow. We will also clarify the geometrical meaning of the supercharges.

\section{$2.1 \quad$ A Toy Model}

In this section we design perhaps the simplest path integral, which has many of the basic properties of cohomological field theory.

Consider a compact complex $n$-dimensional space $X$. We pick local coordinates $x^{I}, I=1, \ldots, 2 n$ on $X$. The local complex coordinates on $X$ will be denoted as $z^{i}, i=1, \ldots, n$; their complex conjugates are $z^{\bar{i}}=\overline{z^{i}}$. Let $X^{I}$ be local coordinates fields describing the position of an instanton on $X$. More precisely, the $X^{I}$ parameterize a map

$$
X^{I}: \text { point } \rightarrow X
$$

We denote by $X^{i}$ local complex coordinates fields and $X^{\bar{i}}$ be their complex conjugates. We call $X^{i}$ and $X^{\bar{i}}$ bosonic fields. We introduce anti-commuting operators $s$ and $\bar{s}$ called supercharges satisfying the following anti-commutation relations,

$$
s^{2}=0, \quad\{s, \bar{s}\}=0, \quad \bar{s}^{2}=0 .
$$

We define a pair of graded quantum number (ghost numbers) $(p, q)$ such that $s$ and $\bar{s}$ carry the following ghost numbers

$$
s:(1,0), \quad \bar{s}:(0,1) .
$$

We call the supersymmetry $(2.2)$ of type $N_{c}=(2,0)$, meaning that we have two supercharges both carrying positive ghost numbers.

We assume that the $X^{i}$ are holomorphic fields, meaning that $\bar{s} X^{i}=$ 0 , and their complex conjugate $X^{\bar{i}}$ are anti-holomorphic, $s X^{\bar{i}}=0$. 
Then we can postulate the following supersymmetry transformation laws

$$
\begin{array}{ll}
s X^{i}=i \psi^{i}, & s \psi^{i}=0 \\
\bar{s} X^{i}=0, & \bar{s} \psi^{i}=0 \\
s X^{\bar{i}}=0, & s \psi^{\bar{i}}=0 \\
\bar{s} X^{\bar{i}}=i \psi^{\bar{i}}, & \bar{s} \psi^{\bar{i}}=0 .
\end{array}
$$

From the above we may write $s$ and $\bar{s}$ as follows

$$
s=i \psi^{i} \frac{\partial}{\partial X^{i}}, \quad \bar{s}=i \psi^{\bar{i}} \frac{\partial}{\partial X^{\bar{i}}} .
$$

We call the anti-commuting superpartners $\psi^{i}$ and $\psi^{\bar{i}}$ of $X^{i}$ and $X^{\bar{i}}$, respectively, fermionic fields. They carry the ghost numbers $(1,0)$ and $(0,1)$, respectively. In general, a field with ghost number $(p, q)$ is fermionic if $p+q$ is odd while, otherwise, it is bosonic.

Now we consider an action functional $S\left(X^{i}, X^{\bar{i}}, \psi^{i}, \psi^{\bar{i}}\right)$ which is invariant under both of thesupersymmetries with supercharges $s$ and $\bar{s}$. The conditions for supersymmetry $s S=\bar{s} S=0$ together with the anticommutation relations (2.2) imply, due to the Poincare lemma, that $S$ may be written as

$$
S=i s \bar{s} \mathcal{K}\left(X^{i}, X^{\bar{i}}\right),
$$

where $\mathcal{K}$ is a locally defined real functional of $X^{i}$ and $X^{\bar{i}}$. Applying the transformation laws (2.4) we have

$$
S=i\left(\frac{\partial^{2} \mathcal{K}}{\partial X^{i} \partial X^{\bar{j}}}\right) \psi^{i} \psi^{\bar{j}}:=-i \mathcal{K}_{i \bar{j}} \psi^{i} \psi^{\bar{j}}
$$

Now we consider the Feynman path integral of our model. The partition function is defined as integration over the space of all fields weighted by $e^{-S}$,

$$
Z=\int[\mathcal{D} X \mathcal{D} \bar{X} \mathcal{D} \psi \mathcal{D} \bar{\psi}] e^{-S}
$$

In everyday quantum field theory, we usually do not have a well-defined path integral measure though we have well-established rules of doing the path integral at least for the perturbative regime. For our trivial 
quantum field theory the path integral measure is perfectly well-defined. The space of all bosonic fields is a copy of $X$. Thus the path integral is an integral over $X$. We have

$$
Z=\int_{X} \prod_{k, \bar{k}=1}^{n} d X^{k} d X^{\bar{k}} d \psi^{k} d \psi^{\bar{k}} \exp \left(i \mathcal{K}_{i \bar{j}} \psi^{i} \psi^{\bar{j}}\right)
$$

Remark that the path integral measure carries ghost number $(n, n)$, i.e., the ghost number anomaly. In the above evaluation we used the basic fact of integration over Grassmann numbers that the integrand should also carry the net ghost number $(n, n)$ to have a non-vanishing integral. Performing the integral over $\psi^{i}$ and $\psi^{\bar{i}}$, using the law of integral over Grassmannian number, we have

$$
Z=\int_{X} \prod_{k, \bar{k}=1}^{n} d X^{k} d X^{\bar{k}} \operatorname{det}\left(i \mathcal{K}_{i \bar{j}}\right)
$$

Now we compare the properties of our model with the differential geometry of the Kähler manifold $X$. We denote the space of $r$-forms on $X$ by $\Omega^{r}(X)$. We have the exterior derivative

$$
d: \Omega^{r}(X) \rightarrow \Omega^{r+1}(X)
$$

satisfying $d^{2}=0$. For any complex manifold we have decompositions

$$
\Omega^{r}(X)=\bigoplus_{r=p+q} \Omega^{p, q}(X)
$$

of $r$-forms into type $(p, q)$-forms with $p+q=r$. Similarly we have a decomposition $d=\partial+\bar{\partial}$ such that

$$
\partial: \Omega^{p, q}(X) \rightarrow \Omega^{p+1, q}(X), \quad \bar{\partial}: \Omega^{p, q}(X) \rightarrow \Omega^{p+1, q}(X),
$$

and

$$
\bar{\partial}^{2}=0, \quad\{\partial, \bar{\partial}\}=0, \quad \bar{\partial}^{2}=0 .
$$

In terms of the local complex coordinates $z^{i}$ and $z^{\bar{i}}$ we have

$$
\partial=d z^{i} \frac{\partial}{\partial z^{i}}, \quad \bar{\partial}=d z^{\bar{i}} \frac{\partial}{\partial z^{\bar{i}}} .
$$


A complex manifold is Kähler iff there exists a non-degenerated type $(1,1)$-form $\varpi$ satisfying $d \varpi=0$. A basic fact of the Kähler geometry is that the Kähler metric tensor $g_{i \bar{j}}$ can be written as

$$
g_{i \bar{j}}=\frac{\partial^{2} f}{\partial z^{i} \partial z^{\bar{j}}}
$$

where $f$ is a Kähler potential. The Kähler form $\varpi$ is given by

$$
\varpi=\varpi_{i \bar{j}} d z^{i} \wedge d z^{\bar{j}}=i g_{i \bar{j}} d z^{i} \wedge d z^{\bar{j}}
$$

where $\varpi_{i \bar{j}}=-\varpi_{\bar{j} i}$ while $g_{i \bar{j}}=g_{\overline{j i}}$.

A comparison with our supersymmetric theory leads to the following obvious dictionary

$$
\begin{aligned}
z^{i} \rightarrow X^{i}, & d z^{i} \rightarrow i \psi^{i}, \\
z^{\bar{i}} \rightarrow X^{\bar{i}}, & d z^{\bar{i}} \rightarrow i \psi^{\bar{i}} .
\end{aligned}
$$

Under the above isomorphism the relations (2.12) and (2.13) become (2.2) and (2.5), respectively, such that

$$
\partial \rightarrow s, \quad \bar{\partial} \rightarrow \bar{s}
$$

Also the Kähler form $\varpi$ in (2.15), after identifying $\mathcal{K}$ with a Kähler potential $f$ of $X$, i.e., $\mathcal{K}_{i \bar{j}}=g_{i \bar{j}}$, becomes (minus) our action functional $S$ in (2.7). Now we examine the partition function $Z$ defined by (2.9). It is obvious that, compare with (2.10)

$$
Z=\int_{X} e^{\varpi}=\int_{X} \frac{\varpi^{n}}{n !}=\int_{X} \prod_{k, \bar{k}=1}^{n} d z^{k} d z^{\bar{k}} \operatorname{det}\left(i g_{i \bar{j}}\right)
$$

where the second identity follows from the fact that the integrand should be a top form and the third identity follows from the definition of $\varpi$. Thus the partition function of our first supersymmetric field theory is the symplectic volume of $X$. We remark that the second identity is equivalent to the condition of the ghost number anomaly cancellation.

One may formalize the above correspondence as follows. For the tangent bundle $T X$ we define an associated superspace $\widehat{T X}$ where the hat symbol denotes the parity change of the fiber as in (2.16). Then 
the supercharges $s$ and $\bar{s}$ are odd vectors and the action $S$ is a function on $\widehat{T X}$.

Now we move on to observables and correlation functions. A supersymmetric observable $\widehat{\alpha}$ is a quantity invariant under the symmetry of the theory and annihilated by supercharges. We consider the following polynomial function on $\widehat{T X}$,

$$
\widehat{\alpha}^{p, q}=\alpha_{i_{1} \ldots i_{p} \bar{j}_{1} \ldots \bar{j}_{q}} \psi^{i_{1}} \ldots \psi^{i_{p}} \psi^{\bar{j}_{1}} \ldots \psi^{\bar{j}_{p}}
$$

carrying the ghost numbers $(p, q)$. Due to the isomorphism (2.16) $\bar{s} \widehat{\alpha}^{p, q}=0$ iff $\bar{\partial} \alpha^{p, q}=0$ where $\alpha^{p, q} \in \Omega^{p, q}(X)$ is the $(p, q)$-form on $X$ defined by

$$
\alpha^{p, q}=\alpha_{i_{1} \ldots i_{p} \bar{j}_{1} \ldots \bar{j}_{q}} d z^{i_{1}} \wedge \ldots \wedge d z^{i_{p}} \wedge d z^{\bar{j}_{1}} \wedge \ldots \wedge d z^{\bar{j}_{p}} .
$$

Note that $\bar{s}$ defines a Dolbeault cohomology on the space of observables graded by the ghost numbers which correspond to the form degrees. In the above we showed that the $\overline{\boldsymbol{s}}$ cohomology is isomorphic to the Dolbeault cohomology $\left(\bar{\partial}, \Omega^{*, *}(X)\right)$ on $X$.

The correlation function of observables or the expectation value is defined by

$$
\left\langle\prod_{m=1}^{r} \widehat{\alpha}^{p_{m}, q_{m}}\right\rangle=\int[\mathcal{D} X \mathcal{D} \bar{X} \mathcal{D} \psi \mathcal{D} \bar{\psi}] \prod_{m=1}^{r} \widehat{\alpha}^{p_{m}, q_{m}} \cdot e^{-S} .
$$

For the present model we see that

$$
\left\langle\prod_{m=1}^{r} \widehat{\alpha}^{p_{m}, q_{m}}\right\rangle=\int_{X} \alpha^{p_{1}, q_{2}} \wedge \ldots \wedge \alpha^{p_{r}, q_{r}} \wedge e^{\varpi} .
$$

Obviously we have non-vanishing correlation function if the observables satisfy the ghost number anomaly cancellation condition

$$
\sum_{m=1}^{r}\left(p_{m}, q_{m}\right)=(\ell, \ell), \quad \ell \leq n
$$

Then

$$
\left\langle\prod_{m=1}^{r} \widehat{\alpha}^{p_{m}, q_{m}}\right\rangle=\frac{1}{(n-\ell) !} \int_{X} \alpha^{p_{1}, q_{2}} \wedge \ldots \wedge \alpha^{p_{r}, q_{r}} \wedge \varpi \wedge \ldots \wedge \varpi .
$$


It follows that correlation functions of supersymmetric observables depend only on the cohomology classes of observables and the Kähler form $\varpi .4$ Thus the correlation function computes the classical cohomology ring of the target space $X$. Equivalently the correlation function computes intersection numbers of homology cycles dual to $\alpha^{p, q} \in H^{p, q}(X)$.

Using our toy model we illustrated many of the basic properties of cohomological field theory. In general, however, life is never as simple as in the idealized world. Typically we encounter an infinite dimensional space of certain set of fields on a manifold $M$ as our target space $X$. Furthermore there usually exists an infinite dimensional group action on the target space. Nonetheless one is eventually interested in the subspace defined as the solution space of certain first order differential equations, modulo the gauge symmetry. Thus we will need a machinery to reduce the path integral to such a subspace and to take care of the group action, as we will do later.

For the time being we ignore those things and assume that the path integral is eventually reduced to some finite dimensional moduli space. Then it may be equivalent to our toy model. We may call a quantum field theory on $M$ with such a property a cohomological field theory. Usually the differential geometrical structures of the moduli space are induced from those of $M$. Such a field theory on $M$ has global supersymmetry equivalent to $(0+0)$-dimensional supersymmetry. The cohomology of such a global supersymmetry is isomorphic to a certain cohomology of $M$. Consequently the correlation functions of supersymmetric observables are differential topological invariant of $M$. We refer to the original paper [1] of Witten for a lucid exposition of general properties of such a cohomological field theory. Here we repeated many of his arguments, perhaps in a slightly different context.

\section{$2.2 \quad N_{c}=(2,2)$ Model}

In this section we consider a somewhat more interesting model by generalizing the toy model of the previous section. We introduce two copies

\footnotetext{
${ }^{4}$ Consider the integral $\int_{X} \beta e^{\varpi}$ where $\beta$ is a closed $(\ell, \ell)$-form. Let $\gamma$ be homology cycle Poincaré dual to $\varpi^{\ell}$. Then the integral reduces to $\frac{1}{(n-\ell) !} \int_{\gamma} \beta$. Let $\beta^{\prime}$ belongs to the same cohomology class as $\beta$, i.e., $\beta^{\prime}=\beta+d \alpha$. We have, using Stokes' theorem, $\int_{\gamma}\left(\beta^{\prime}-\beta\right)=\int_{\gamma} d \alpha=\int_{\partial \gamma} \alpha=0$.
} 
$\left(s_{ \pm}, \bar{s}_{ \pm}\right)$of the fermionic charges $(s, \bar{s})$. We regard the above doubling as a $Z_{2}$-grading in the sense that supercharges carry the following ghost numbers $(p, q)$ introduced for the toy model,

$$
\begin{array}{ll}
\boldsymbol{s}_{+}:(+1,0), & \overline{\boldsymbol{s}}_{+}:(0,+1), \\
\boldsymbol{s}_{-}:(-1,0), & \overline{\boldsymbol{s}}_{-}:(0,-1) .
\end{array}
$$

Thus the supercharges $\boldsymbol{s}_{+}$and $\overline{\boldsymbol{s}}_{+}$can be identified with the original supercharges $s$ and $\bar{s}$ of the toy model. We want to define a supersymmetric theory invariant under all four supercharges. Obviously we will have a $Z_{2}$-symmetry exchanging the + and - indices. We will say that the resulting theory is of type $N_{c}=(2,2)$. We will see that such a model is related with the geometry of tangent bundle $T X$ of a Kähler manifold $X$. The partition function of this model can be identified with the Euler characteristic of $X$.

\subsubsection{Basic Structures}

We postulate that the supercharges satisfy the following anti-commutation relations,

$$
s_{ \pm}^{2}=0, \quad\left\{s_{ \pm}, \bar{s}_{ \pm}\right\}=0, \quad \bar{s}_{ \pm}^{2}=0,
$$

and

$$
\left\{\boldsymbol{s}_{+}, \boldsymbol{s}_{-}\right\}=0, \quad\left\{\boldsymbol{s}_{ \pm}, \overline{\boldsymbol{s}}_{\mp}\right\}=0, \quad\left\{\overline{\boldsymbol{s}}_{+}, \overline{\boldsymbol{s}}_{-}\right\}=0,
$$

which is an obvious generalization of (2.2). We will consider the same bosonic fields $X^{i}$ and $X^{\bar{i}}$ as in our toy model. We demand $X^{i}$ to be biholomorphic or chiral, meaning that $\bar{s}_{ \pm} X^{i}=0 .{ }^{5}$ We call the complex conjugates $X^{\bar{i}}$ anti-chiral, meaning that $s_{+} X^{\bar{i}}=s_{-} X^{\bar{i}}=0$. Now the anti-commutation relations among supercharges suggest that we have the following chiral multiplets

$$
\psi_{-}^{i} \underset{s_{+} \downarrow}{\stackrel{s_{-}}{\longleftarrow}} X^{i} \stackrel{s_{+}}{\longleftrightarrow} H_{+}^{i}{ }^{i} .
$$

${ }^{5}$ Note that this choice is arbitrary. We may also demand twisted bi-holomorphicity or twisted chirality by imposing $\bar{s}_{+} X^{i}=s_{-} X^{i}=0$. A model with both chiral and twisted chiral multiplets has very interesting properties. 
In the above $H^{i}$ are called auxiliary fields, which are introduced due to the conditions

$$
\left\{\boldsymbol{s}_{+}, \boldsymbol{s}_{-}\right\} X^{i}=i \boldsymbol{s}_{+} \psi_{-}^{i}+i \boldsymbol{s}_{-} \psi_{+}^{i}=0,
$$

can be solved as $s_{ \pm} \psi^{i}= \pm H^{i}$ while they are indeterminate. ${ }^{6}$ Denoting $\delta=s_{+} \bar{\epsilon}_{-}+s_{-} \bar{\epsilon}_{+}+\bar{s}_{+} \epsilon_{-}+\bar{s}_{-} \epsilon_{+}$we have the following transformation laws for chiral and anti-chiral multiplets,

$$
\begin{aligned}
& \delta X^{i}=i \bar{\epsilon}_{-} \psi_{+}^{i}+i \bar{\epsilon}_{+} \psi_{-}^{i}, \quad \delta X^{\bar{i}}=i \epsilon_{-} \psi_{+}^{\bar{i}}+i \epsilon_{+} \psi_{-}^{\bar{i}}, \\
& \delta \psi_{+}^{i}=+\bar{\epsilon}_{+} H^{i}, \quad \delta \psi_{+}^{\bar{i}}=+\epsilon_{+} H^{i}, \\
& \delta \psi_{-}^{i}=-\bar{\epsilon}_{-} H^{i}, \quad \delta \psi_{-}^{\bar{i}}=-\epsilon_{-} H^{\bar{i}}, \\
& \delta H^{i}=0 \quad \delta H^{\bar{i}}=0 .
\end{aligned}
$$

Now we define a natural supersymmetric action functional. The requirements $s_{ \pm} S=\overline{\boldsymbol{s}}_{ \pm} S=0$ for $S$ to have $N_{c}=(2,2)$ supersymmetry and the anti-commutation relations (2.26) and eqrefabac imply, by repeatedly applying the Poincaré lemma, that we can write $S$ as follows,

$$
S=s_{+} \bar{s}_{+} s_{-} \bar{s}_{-} \mathcal{K}\left(X^{i}, X^{\bar{i}}\right),
$$

where $\mathcal{K}\left(X^{i}, X^{\bar{i}}\right)$ is a locally defined real functional. Expanding the above we have

$$
S=g_{i \bar{j}} H^{i} H^{\bar{j}}+i \partial_{k} g_{i \bar{j}} \psi_{+}^{k} \psi_{-}^{i} H^{\bar{j}}+i \partial_{\bar{k}} g_{i \bar{j}} \psi_{+}^{\bar{k}} H^{i} \psi_{-}^{\bar{j}}+\partial_{\ell} \partial_{\bar{k}} g_{i \bar{j}} \psi_{+}^{\ell} \psi_{+}^{\bar{k}} \psi_{-}^{i} \psi_{-}^{\bar{j}},
$$

where $\partial_{i}=\partial / \partial X^{i}$ and $\partial_{\bar{j}}=\partial / \partial X^{\bar{j}}$ and we set $g_{i \bar{j}}:=\partial_{i} \partial_{\bar{j}} \mathcal{K}$. We can integrate out the auxiliary fields $H^{i}$ and $H^{\bar{i}}$ by a Gaussian integral, or, equivalently, eliminate them by pluging in the algebraic equations of motions for $H^{i}$ and $H^{\bar{i}}$;

$$
\begin{aligned}
& H^{i}=-i g^{i \bar{j}} \partial_{k} g_{\ell \bar{j}} \psi_{+}^{k} \psi_{-}^{\ell}, \\
& H^{\bar{j}}=+i g^{i \bar{j}} \partial_{\bar{k}} g_{i \bar{\ell}} \psi_{+}^{\bar{k}} \psi_{-}^{\bar{\ell}},
\end{aligned}
$$

where $g^{i \bar{j}}$ is the inverse of $g_{i \bar{j}}$. Then we obtain the new action functional $S^{\prime}$,

$$
S^{\prime}=-R_{\ell \bar{k} i \bar{j}} \psi_{+}^{\ell} \psi_{+}^{\bar{k}} \psi_{-}^{i} \psi_{-}^{\bar{j}},
$$

\footnotetext{
${ }^{6}$ The equation might also be solved as $s_{ \pm} \psi^{i}=0$ without introducing $H^{i}$. However, the auxiliary fields are indispensable. The moral is that we better keep it whenever we encounter redundancy.
} 
where

$$
R_{\ell \bar{k} i \bar{j}}=-\partial_{\ell} \partial_{\bar{k}} g_{i \bar{j}}+g^{p \bar{q}} \partial_{i} g_{p \bar{k}} \partial_{\bar{j}} g_{\ell \bar{q}}
$$

which can be identified with the the Riemann curvature tensor of $T X$ if $\mathcal{K}$ is a Kähler potential of $X$. Remark that the non-vanishing components of the Christoffel symbols in the Kähler geometry are

$$
\Gamma_{k \ell}^{i}=g^{i \bar{j}} \partial_{k} g_{\ell \bar{j}}, \quad \Gamma_{\overline{k \ell}}^{\bar{i}}=g^{i \bar{j}} \partial_{\bar{k}} g_{i \bar{\ell}} .
$$

The new action $S^{\prime}$ is invariant under the supersymmetry after modifying the transformation laws (2.30) by replacing $H^{i}$ and $H^{\bar{i}}$ by their on-shell expressions (2.33).

Now we examine the path integral. The partition function is defined as usual,

$$
\begin{aligned}
Z & =\int\left[\mathcal{D} X \mathcal{D} \bar{X} \mathcal{D} \psi_{ \pm} \mathcal{D} \bar{\psi}_{ \pm}\right] e^{-S^{\prime}} \\
& =\left(\frac{1}{2 \pi}\right)^{n} \int \prod_{k, \bar{k}=1}^{n} d X^{k} d X^{\bar{k}} d \psi_{+}^{k} d \psi_{+}^{\bar{k}} d \psi_{-}^{k} d \psi_{-}^{\bar{k}} \exp \left(R_{\ell \bar{k} i \bar{j}} \psi_{+}^{\ell} \psi_{+}^{\bar{k}} \psi_{-}^{i} \psi_{-}^{\bar{j}}\right)
\end{aligned}
$$

where the integration is over the space of all fields. The bosonic part of the path integral is an integration over a copy of $X$. We first perform the integral over $\psi_{+}^{i}$ and $\psi_{+}^{\bar{i}}$ which, as we saw earlier, is equivalent to replacing $R_{\ell \bar{k} i \bar{j}} \psi_{+}^{\ell} \psi_{+}^{\bar{k}} \psi_{-}^{i} \psi_{-}^{\bar{j}}$ by the $(1,1)$-form $\boldsymbol{R}_{i \bar{j}}:=R_{k \bar{\ell} i \bar{j}} d z^{k} \wedge d \bar{z}^{\bar{\ell}}$ on $X$,

$$
Z=\left(\frac{1}{2 \pi}\right)^{n} \int_{X} \prod_{k, \bar{k}=1}^{n} d \psi_{-}^{k} d \psi_{-}^{\bar{k}} \exp \left(\boldsymbol{R}_{i \bar{j}} \psi_{-}^{i} \psi_{-}^{\bar{j}}\right)
$$

Integration over $\psi_{-}^{i}$ and $\psi^{\bar{i}}$ leads to

$$
Z=\frac{1}{(2 \pi)^{n}} \int_{X} \operatorname{det}\left(\boldsymbol{R}_{i \bar{j}}\right)=\int_{X} e(T X):=\chi(T X)
$$

The last identity is due to the Gauss-Bonnet theorem. Thus the partition function computes the Euler characteristic $\chi(X)=\chi(T X)$ of the manifold $X$. 


\subsubsection{Geometrical Interpretation of Supercharges}

Now we examine the geometrical meaning of our supercharges. In Sect. 2.1.1 we already saw that the supercharges $s_{+}$and $\overline{\boldsymbol{s}}_{+}$are associated with the $\partial$ and $\bar{\partial}$ differential on the target space $X$. Our task is to understand the geometrical meaning of the remaining supercharges $s_{-}$ and $\bar{s}_{-}$.

We begin with discarding the obvious candidates for $\boldsymbol{s}_{-}$and $\overline{\boldsymbol{s}}_{-}$, namely the operators $\partial^{*}$ and $\bar{\partial}^{*}$ defined by

$$
\begin{aligned}
& \partial^{*}=-* \bar{\partial} *: \Omega^{p, q}(X) \rightarrow \Omega^{p-1, q}(X), \\
& \bar{\partial}^{*}=-* \partial *: \Omega^{p, q}(X) \rightarrow \Omega^{p, q-1}(X),
\end{aligned}
$$

where $*$ denote the Hodge star. They satisfy the following relations

$$
\partial^{* 2}=0, \quad\left\{\partial^{*}, \bar{\partial}^{*}\right\}=0, \quad \bar{\partial}^{* 2}=0,
$$

and decrease the form degree by $(-1,0)$ and $(0,-1)$, respectively. We have, however, well-known relations in Kähler geometry

$$
\left\{\partial, \partial^{*}\right\}=\left\{\bar{\partial}, \bar{\partial}^{*}\right\}=\frac{1}{2}\left\{d, d^{*}\right\}=\frac{1}{2} \nabla,
$$

where $\nabla$ is the Laplacian. On the other hand we have $\left\{s_{+}, s_{-}\right\}=$ $\left\{\bar{s}_{+}, \bar{s}_{-}\right\}=0$. We also have more obvious problem from $\partial^{*} X^{i}=0$, while $s_{-} X^{i}=i \psi_{-}^{i} \neq 0$. Thus we have to seek an alternative set of operators.

We first consider the real symplectic case and then specialize to the Kähler case. Consider a symplectic manifold with symplectic form $\varpi=$ $\varpi_{I J} d x^{I} \wedge d x^{J}$. Since the matrix $\varpi_{I J}=-\varpi_{J I}$ is non-degenerated we have a well-defined inverse matrix $\varpi^{J I}$. Using $\varpi^{J I}$ we have a canonical map from a cotangent vector to a tangent vector. ${ }^{7}$ Denoting $\alpha=\alpha_{I} d x^{I}$ and $\widetilde{\alpha}=\widetilde{\alpha}^{I} \frac{\partial}{\partial x^{I}}$ for a cotangent vector and its dual tangent vector, respectively, we have

$$
\widetilde{\alpha}^{I}=\varpi^{I J} \alpha_{J}
$$

One may define the corresponding operator $\sqcap$ as follows

$$
\sqcap:=\frac{\varpi^{I J}}{2}\left(\left(\otimes \frac{\partial}{\partial x^{I}}\right) \frac{\partial}{\partial\left(d x^{J}\right)}-\left(\otimes \frac{\partial}{\partial x^{J}}\right) \frac{\partial}{\partial\left(d x^{I}\right)}\right),
$$

\footnotetext{
${ }^{7}$ We may also consider a Poisson manifold with a bi-vector $\varpi^{I J}$.
} 
where the symbol $\otimes \frac{\partial}{\partial x^{I}}$ means taking tensor product. For instance we have

$$
\sqcap \alpha=\varpi^{I J} \alpha_{L}\left(\otimes \frac{\partial}{\partial x^{I}}\right) \frac{\partial\left(d x^{L}\right)}{\partial\left(d x^{J}\right)}=\varpi^{I J} \alpha_{J} \frac{\partial}{\partial x^{I}}=\widetilde{\alpha}^{I} \frac{\partial}{\partial x^{I}}=\widetilde{\alpha} .
$$

Similarly $\sqcap$ induce an isomorphism

$$
\sqcap: \Gamma\left(\wedge^{p} T^{*} X \otimes \wedge^{q} T X\right) \rightarrow \Gamma\left(\wedge^{p-1} T^{*} X \otimes \wedge^{q+1} T X\right),
$$

where $T X$ and $T^{*} X$ are the tangent and cotangent vector, respectively, and $\Gamma$ denotes the space of sections. Note that $\Gamma\left(\wedge^{p} T^{*} X \otimes \wedge^{q} T X\right)=$ $\Omega^{p}\left(X, \wedge^{q} T X\right)$.

Now we can define a first order differential operator by taking the composition of $\sqcap$ and the exterior derivative $d$,

$$
d: \Omega\left(\wedge^{p} T^{*} X \otimes \wedge^{q} T X\right) \rightarrow \Omega\left(\wedge^{p+1} T^{*} X \otimes \wedge^{q} T X\right),
$$

as follows,

$$
\tilde{d}:=(\sqcap d-d \sqcap): \Omega\left(\wedge^{p} T^{*} X \otimes \wedge^{q} T X\right) \rightarrow \Omega\left(\wedge^{p} T^{*} X \otimes \wedge^{q+1} T X\right) .
$$

We will conveniently assign the form degree -1 to the operator $\tilde{d}$. One can check

$$
\widetilde{d}^{2}=0, \quad\{d, \widetilde{d}\}=0,
$$

after a direct computations. We also have the following obvious but important relation

$$
\begin{aligned}
& d: x^{I} \rightarrow d x^{I} \\
& \tilde{d}: x^{I} \rightarrow \varpi^{I J} \frac{\partial}{\partial x^{I}}
\end{aligned}
$$

Thus for a symplectic manifold $X$ with the symplectic form $\varpi$ we have

$$
\left(x^{I}, d x^{I}, \partial / \partial x^{I} ; d, \widetilde{d}\right)
$$

where $d x^{I}$ and $\partial / d x^{I}$ denote local coordinates in the fiber of $T X$ and the fiber of $T^{*} X$, respectively. To relate with supersymmetry we perform the parity changes for both the fibers of $T X$ and $T^{*} X$, i.e., $\overline{T X}$ and $\widehat{T^{*} X}$. Then we have a map

$$
\left(x^{I}, d x^{I}, \partial / \partial x^{I} ; d, \widetilde{d}\right) \rightarrow\left(X^{I}, i \psi_{+}^{I}, i \chi_{I} ; Q_{+}, Q_{-}\right),
$$


where everything is in real coordinates, $\psi_{-}^{I}:=\varpi^{I J} \chi_{J}$ and $Q_{ \pm}=s_{ \pm}+\bar{s}_{ \pm}$.

One may compare our operator $\tilde{d}$ with the (different) operator $\Delta$ defined by Koszul [26]. The operator $\Delta$ is define as

$$
\Delta:=\sqcap_{k} d-d \sqcap_{k},
$$

where $\Pi_{k}$ in the notation of (2.44) is given by

$$
\Pi_{k}:=\frac{\varpi^{I J}}{2}\left(\frac{\partial^{2}}{\partial\left(d x^{I}\right) \partial\left(d x^{J}\right)}-\frac{\partial^{2}}{\partial\left(d x^{J}\right) \partial\left(d x^{I}\right)}\right) .
$$

Thus $\Delta$ is a second order differential operator with degree -1 on $\Gamma\left(\wedge^{*} T^{*} X\right)=\Omega^{*}(X)$ and we have $\Delta x^{I}=0 .^{8}$

Now we return to a Kähler manifold $X$ with Kähler form $\varpi=$ $\varpi_{i j} d z^{i} \wedge d z^{\bar{j}}$ and show that the above interpretation is indeed the correct one. It is suffice to consider the holomorphic half, say $s_{+}$and $s_{-}$. The operator $\sqcap$ is decomposed as $\Pi=\Pi^{\prime}+\Pi^{\prime \prime}$ where

$$
\begin{aligned}
& \widehat{\Pi}^{\prime}=-\frac{1}{2} \varpi^{i \bar{j}}\left(X^{\ell}, X^{\bar{\ell}}\right) \chi_{\bar{j}} \frac{\partial}{\partial \psi_{+}^{i}}, \\
& \widehat{\Pi}^{\prime \prime}=+\frac{1}{2} \varpi^{i \bar{j}}\left(X^{\ell}, X^{\bar{\ell}}\right) \chi_{i} \frac{\partial}{\partial \psi_{+}^{\bar{j}}}
\end{aligned}
$$

where we did parity change $\sqcap \rightarrow \widehat{\Pi}$ by

$$
\begin{array}{ll}
d z^{i} \rightarrow i \psi_{+}^{i}, & \partial / \partial z^{i} \rightarrow i \chi_{i}, \\
d z^{\bar{i}} \rightarrow i \psi_{+}^{\bar{i}}, & \partial / \partial z^{\bar{i}} \rightarrow i \chi_{i} .
\end{array}
$$

Now we define

$$
s_{-}=\widehat{\Pi}^{\prime} s_{+}-s_{+} \widehat{\Pi}^{\prime}
$$

From

$$
\boldsymbol{s}_{+}=i \psi_{+}^{i} \frac{\partial}{\partial X^{i}}
$$

\footnotetext{
${ }^{8}$ Koszul proved $\Delta^{2}=\{d, \Delta\}=0$ and defined a covariant Schouten-Nijenhuis bracket, $\alpha, \beta \in \Omega^{*}(X)$,

$$
\{\alpha, \beta\}_{S N}=(\Delta \alpha) \wedge \beta+(-1)^{|a|} \alpha \wedge \Delta \alpha-\Delta(\alpha \wedge \beta) .
$$
}


we have

$$
\boldsymbol{s}_{-}=-\frac{i}{2} \varpi^{i \bar{j}} \chi_{\bar{j}} \frac{\partial}{\partial X^{i}}+\frac{i}{2} \frac{\partial \varpi^{i \bar{j}}}{\partial X^{k}} \psi_{+}^{k} \chi_{\bar{j}} \frac{\partial}{\partial \psi_{+}^{i}} .
$$

Now we can check if the above identification of the supercharge $\boldsymbol{s}_{-}$ is the correct one. After direct computations we find the following relations

$$
\begin{array}{ll}
s_{+} X^{i}=i \psi_{+}^{i}, & s_{+} \psi_{+}^{i}=0, \\
s_{+} X^{\bar{i}}=0, & s_{+} \psi_{-}^{i}=+i \varpi_{\bar{j} \ell} \frac{\partial \varpi^{i \bar{j}}}{\partial X^{k}} \psi_{+}^{k} \psi_{-}^{\ell}, \\
s_{-} X^{\bar{i}}=0, & s_{-} \psi_{+}^{i}=-i \varpi_{\bar{j} \ell} \frac{\partial \varpi^{i \bar{j}}}{\partial X^{k}} \psi_{+}^{k} \psi_{-}^{\ell}, \\
s_{-} X^{i}=i \psi_{-}^{i}, & s_{-} \psi_{-}^{i}=0,
\end{array}
$$

where we defined

$$
\psi_{-}^{i}=-\frac{1}{2} \varpi^{i \bar{j}} \chi_{\bar{j}} .
$$

In checking $s_{-} \psi_{-}^{i}=0$ we used the torsion-free condition of the Hermitian connection of $T X$, equivalent to the condition $d \varpi=0$. Using the relation

$$
\varpi_{i \bar{j}}=i g_{i \bar{j}}=-\varpi_{\bar{j} i}
$$

we see that the above is exactly the supersymmetry algebra of $\boldsymbol{s}_{+}$and $s_{-}$in (2.30) after replacing the auxiliary fields $H^{i}$ by their on-shell values given by $(2.33)$.

Now we summarize. We have the following operators

$$
\begin{array}{rlrl}
\boldsymbol{s}_{+} & =i \psi_{+}^{i} \frac{\partial}{\partial X^{i}}, & \boldsymbol{s}_{-} & =\widehat{\Pi}^{\prime} \boldsymbol{s}_{+}-\boldsymbol{s}_{+} \widehat{\Pi}^{\prime}, \\
\overline{\boldsymbol{s}}_{+} & =i \psi_{+}^{\bar{i}} \frac{\partial}{\partial X^{\bar{i}}}, & \overline{\boldsymbol{s}}_{-}=\widehat{\Pi}^{\prime \prime} \overline{\boldsymbol{s}}_{+}-\overline{\boldsymbol{s}}_{+} \widehat{\Pi}^{\prime \prime},
\end{array}
$$

such that

$$
\begin{aligned}
& s_{+}: \widehat{\Omega}^{p, q}\left(\wedge^{r} \widehat{\mathcal{T} X} \otimes \wedge^{s} \widehat{\widehat{\mathcal{T} X}}\right) \rightarrow \widehat{\Omega}^{p+1, q}\left(\wedge^{r} \widehat{\mathcal{T} X} \otimes \wedge^{s} \widehat{\widehat{\mathcal{T} X}}\right) \\
& \bar{s}_{+}: \widehat{\Omega}^{p, q}\left(\wedge^{r} \widehat{\mathcal{T} X} \otimes \wedge^{s} \widehat{\widehat{\mathcal{T} X}}\right) \rightarrow \widehat{\Omega}^{p, q+1}\left(\wedge^{r} \widehat{\mathcal{T} X} \otimes \wedge^{s} \widehat{\widehat{\mathcal{T} X}}\right), \\
& s_{-}: \widehat{\Omega}^{p, q}\left(\wedge^{r} \widehat{\mathcal{T} X} \otimes \wedge^{s} \widehat{\widehat{\mathcal{T}} X}\right) \rightarrow \widehat{\Omega}^{p, q}\left(\wedge^{r+1} \widehat{\mathcal{T} X} \otimes \wedge^{s} \widehat{\widehat{\mathcal{T} X}}\right) \\
& \overline{\boldsymbol{s}}_{-}: \Omega^{p, q}\left(\wedge^{r} \widehat{\mathcal{T} X} \otimes \wedge^{s} \widehat{\widehat{\mathcal{T} X}}\right) \rightarrow \widehat{\Omega}^{p, q}\left(\wedge^{r} \widehat{\mathcal{T} X} \otimes \wedge^{s+1} \widehat{\widehat{\mathcal{T} X}}\right)
\end{aligned}
$$


where $\mathcal{T} X$ denotes the holomorphic parts of the tangent bundle $T X=$ $\mathcal{T} X \oplus \overline{\mathcal{T}} X$ of $X$

\subsubsection{Introducing a Holomorphic Potential}

Now we consider a more general action functional. We pick a holomorphic function $\mathcal{W}\left(X^{i}\right)$ of the chiral fields $X^{i}$. Since $\bar{s}_{ \pm} X^{i}=0$ we have $\bar{s}_{ \pm} \mathcal{W}\left(X^{i}\right)=0$. It follows that we have the following more general $N=(2,2)$ supersymmetric action functional,

$$
S(\lambda)=s_{+} \bar{s}_{+} s_{-} \bar{s}_{-} \mathcal{K}\left(X^{i}, X^{\bar{i}}\right)+\lambda s_{+} s_{-} \mathcal{W}\left(X^{i}\right)+\lambda \bar{s}_{+} \bar{s}_{-} \overline{\mathcal{W}}\left(X^{i}\right),
$$

where $\lambda$ is certain coupling constant introduced for convenience. Expanding $S(\lambda)$ we find

$$
\begin{aligned}
S(\lambda)= & g_{i \bar{j}} H^{i} H^{\bar{j}}+i\left(\partial_{k} g_{i j} \psi_{+}^{k} \psi_{-}^{i}-\lambda V_{\bar{j}}\right) H^{\bar{j}}+i H^{i}\left(\partial_{\bar{k}} g_{i \bar{j}} \psi_{+}^{\bar{k}} \psi_{-}^{\bar{j}}-\lambda V_{i}\right) \\
& -\lambda \frac{\partial V_{i}}{\partial X^{j}} \psi_{+}^{i} \psi_{-}^{j}-\lambda \frac{\partial V_{\bar{i}}^{\bar{i}}}{\partial X^{\bar{j}}} \psi_{+}^{\bar{i}} \psi_{-}^{\bar{j}}+\partial_{\ell} \partial_{\bar{k}} g_{i \bar{j}} \psi_{+}^{\ell} \psi_{+}^{\bar{k}} \psi_{-}^{i} \psi_{-}^{\bar{j}} .
\end{aligned}
$$

where we set $V_{i}:=\partial \mathcal{W} / \partial X^{i}$. Now we integrate out the auxiliary fields by their algebraic equations of motions

$$
\begin{aligned}
& H^{i}=-i \Gamma_{k \ell}^{i} \psi_{+}^{k} \psi_{-}^{\ell}+i \lambda g^{i \bar{j}} V_{\bar{j}}, \\
& H^{\bar{i}}=+i \Gamma_{\bar{k} \ell}^{\bar{i}} \psi_{+}^{\bar{k}} \psi_{-}^{\bar{\ell}}-i \lambda g^{j \bar{i}} V_{j},
\end{aligned}
$$

where we used the notations in (2.36). We have

$$
\begin{gathered}
S^{\prime}(\lambda)=\lambda^{2} g^{i \bar{j}} V_{i} V_{\bar{j}}-\lambda \frac{D V_{i}}{D X^{j}} \psi_{+}^{i} \psi_{-}^{j}-\lambda \frac{D V_{\bar{i}}^{\bar{i}}}{D X^{\bar{j}}} \psi_{+}^{\bar{i}} \psi_{-}^{\bar{j}} \\
-R_{\ell \bar{k} i \bar{j}} \psi_{+}^{\ell} \psi_{+}^{\bar{k}} \psi_{-}^{i} \psi_{-}^{\bar{j}} .
\end{gathered}
$$

where

$$
\frac{D V_{i}}{D X^{j}}:=\frac{\partial V_{i}}{\partial X^{j}}+\Gamma_{i j}^{\ell} V_{\ell}
$$

\subsubsection{The Partition Function}

The partition function is independent of $\lambda$ since $\lambda$ dependent term is $\boldsymbol{s}_{ \pm^{-}}$ exact deformation of $S$. In the limit $\lambda \rightarrow \infty$ the dominant contributions 
to the path integral are from the vanishing locus of holomorphic vector fields $V_{i}$. Or we may simply apply the fixed point theorem of Witten to reach the same conclusion; from the supersymmetry transformation laws (2.30) we see that the fixed point equations are $\psi_{ \pm}^{\bar{j}}=H^{\bar{i}}=0$. From the relations (2.41) the above implies $V_{i}=0$.

For generic choices the vanishing locus will be zero dimensional and consists of isolated points. Then there are no fermionic zero-modes and the action functional evaluated at such a point is simply 0 . Thus the partition function is just the sum of contributions of each point weighted by the one loop determinants of the transverse degrees of freedom. Due to the Bose-Fermi symmetry such a determinant is \pm 1 , depending on a certain orientation, due to supersymmetry and due to the ambiguity in taking the square root of the determinant. In our case they always can be set +1 since the ambiguities from holomorphic and anti-holomorphic contribution cancel each other. Thus the partition function is the number of zeros. If we turn off the potential we recover the original model. This gives rise to the Poincaré-Hopf theorem. We should mention that the usual derivation of Poincaré-Hopf theorem uses supersymmetric quantum mechanics, i.e., the $(0+1)$ dimensional sigma model [27], [28], [20], but with essentially the same arguments.

For a non-generic vector field $V_{i}$ the vanishing locus can be a positive dimensional submanifold. One may try to perturb the vector field $V_{i}$, thus $\mathcal{W}\left(X^{i}\right)$, to a generic one or just evaluate the path integral. We will give a detailed analysis for this case in the next section in a more general context.

\subsection{Generalization to $N_{c}=(2,0)$ Model}

The model in the previous section enjoys a perfect symmetry between things with + and - indices. Now we want to relax such a symmetry. We shall see that such symmetry is due to the restriction of considering a very special Hermitian holomorphic vector bundle, namely the tangent bundle $T X$, over $X$. By maintaining only the $N_{c}=(2,0)$ supersymmetry generated by $s_{+}$and $\bar{s}_{+}$we arrive at a more general model, which is related with a Hermitian holomorphic bundle $\mathbb{E}$ over $X$. 


\subsubsection{The Basic Structures}

First we write our action functional $S(\lambda)$ (2.64) in form such that only the $s_{+}$and $\bar{s}_{+}$are manifest,

$$
\begin{aligned}
S(\lambda)= & -s_{+} \bar{s}_{+}\left(g_{i \bar{j}}\left(X^{i}, X^{\bar{i}}\right) \psi_{-}^{i} \psi_{-}^{\bar{j}}\right)+i \lambda s_{+}\left(\psi_{-}^{i} V_{i}\left(X^{j}\right)\right) \\
& +i \lambda \bar{s}_{+}\left(\psi_{-}^{\bar{i}} V_{\bar{i}}\left(X^{\bar{j}}\right)\right) .
\end{aligned}
$$

Similarly we disconnect the diagram (2.28) by removing the link $s_{-}$

$$
\begin{array}{lll}
\psi_{-}^{i} \underset{s_{+} \searrow}{ }{ } X^{i} \stackrel{s_{+}}{\longrightarrow} \psi_{+}^{i} \\
\end{array}
$$

Now we can regard the above as two independent sets of multiplets. Then we rename various fields as follows

$$
\begin{array}{ll}
\psi_{-}^{i} \rightarrow \chi_{-}^{\alpha}, \quad H^{i} \rightarrow H^{\alpha}, \quad V_{i} \rightarrow \mathfrak{S}_{\alpha}\left(X^{j}\right), \quad g_{i \bar{j}} \rightarrow h_{\alpha \bar{\beta}}\left(X^{i}, X^{\bar{i}}\right), \\
\psi_{-}^{\bar{i}} \rightarrow \chi_{-}^{\bar{\alpha}}, \quad H^{\bar{i}} \rightarrow H^{\bar{\alpha}}, \quad V_{\bar{i}} \rightarrow \mathfrak{S}_{\bar{\alpha}}\left(X^{\bar{j}}\right),
\end{array}
$$

where the new indices run as $\alpha, \beta=1, \ldots, r$ and we maintain the Hermiticity of $h_{\alpha \bar{\beta}}$. The $s_{+}$and $\bar{s}_{+}$transformation laws are

$$
\begin{array}{ll}
\delta X^{i}=i \bar{\epsilon}_{-} \psi_{+}^{i}, \quad & \delta \psi_{+}^{i}=0 \\
\delta X^{\bar{i}}=i \epsilon_{-} \psi_{+}^{\bar{i}}, & \delta \psi_{+}^{\bar{i}}=0
\end{array}
$$

and

$$
\begin{array}{ll}
\delta \chi_{-}^{\alpha}=-\bar{\epsilon}_{-} H^{\alpha}, & \delta H^{\alpha}=0 \\
\delta \chi_{-}^{\bar{\alpha}}=-\epsilon_{-} H^{\bar{\alpha}}, & \delta H^{\bar{\alpha}}=0 .
\end{array}
$$

Now we have following new action functional

$$
\begin{aligned}
S= & -s_{+} \bar{s}_{+}\left(h_{\alpha \bar{\beta}}\left(X^{i}, X^{\bar{i}}\right) \chi_{-}^{\alpha} \chi_{-}^{\bar{\beta}}\right)+i s_{+}\left(\chi_{-}^{\alpha} \mathfrak{S}_{\alpha}\left(X^{i}\right)\right) \\
& +i \bar{s}_{+}\left(\chi_{-}^{\bar{\alpha}} \mathfrak{S}_{\bar{\alpha}}\left(X^{\bar{i}}\right)\right)
\end{aligned}
$$

which is the general form of $N_{c}=(2,0)$ supersymmetric action functional. 
Note that the above action functional may or may not have $N_{c}=$ $(2,2)$ symmetry. Generically the model does not have $N_{c}=(2,2)$ supersymmetry. Note also that the model has the same supersymmetry as our toy model in Sect. 2.1.1. Thus the new model shares the same observables with the toy models, which are $\widehat{\alpha}^{p, q}$ obtained by an element $\alpha^{p, q}=H^{p, q}(X)$ of the cohomology group $H^{p, q}(X)$ after the parity change $T X \rightarrow \widehat{T X}$. The differences with the toy model are that we have additional Fermi multiplets $\left(\chi_{-}^{\alpha}, H^{\alpha}\right)$ with a different action functional. We call the multiplets $\left(\chi_{-}^{\alpha}, H^{\alpha}\right)$ Fermi multiplets. We call $\chi_{-}^{\alpha}$ anti-ghosts. We remark that the action functional of the toy model may be regarded as zero by treating the Kähler form $\varpi$ as an observables. Now we turn to examine the action functional.

Expanding $S$ we have

$$
\begin{aligned}
S= & h_{\alpha \bar{\beta}} H^{\alpha} H^{\bar{\beta}}+i\left(\partial_{i} h_{\alpha \bar{\beta}} \psi_{-}^{i} \chi_{+}^{\alpha}-\mathfrak{S}_{\bar{\beta}}\right) H^{\bar{\beta}}+i H^{\alpha}\left(\partial_{\bar{j}} h_{\alpha \bar{\beta}} \psi_{+}^{\bar{j}} \chi_{-}^{\bar{\beta}}-\mathfrak{S}_{\alpha}\right) \\
& -\frac{\partial \mathfrak{S}_{\alpha}}{\partial X^{j}} \psi_{+}^{j} \chi_{-}^{\alpha}-\frac{\partial \mathfrak{S}_{\bar{\alpha}}}{\partial X^{\bar{j}}} \psi_{+}^{\bar{j}} \chi_{-}^{\bar{\alpha}}+\left(\partial_{i} \partial_{\bar{j}} h_{\alpha \bar{\beta}}\right) \psi_{+}^{i} \psi_{+}^{\bar{j}} \chi_{-}^{\alpha} \chi_{-}^{\bar{\beta}} .
\end{aligned}
$$

After integrating out the auxiliary fields $H^{\alpha}$ and $H^{\bar{\beta}}$ by their algebraic equations of motion

$$
\begin{aligned}
& H^{\alpha}=-i h^{\alpha \bar{\beta}} \partial_{k} h_{\gamma \bar{\beta}} \psi_{+}^{k} \chi_{-}^{\gamma}+i h^{\alpha \bar{\beta}} \mathfrak{S}_{\bar{\beta}}, \\
& H^{\bar{\alpha}}=+i h^{\alpha \bar{\beta}} \partial_{\bar{k}} h_{\alpha \bar{\gamma}} \psi_{+}^{\bar{k}} \chi_{-}^{\bar{\gamma}}-i h^{\beta \bar{\alpha}} \mathfrak{S}_{\beta},
\end{aligned}
$$

we are left with

$$
S^{\prime}=h^{\alpha \bar{\beta}} \mathfrak{S}_{\alpha} \mathfrak{S}_{\bar{\beta}}-\frac{D \mathfrak{S}_{\alpha}}{D X^{j}} \psi_{+}^{j} \chi_{-}^{\alpha}-\frac{D \mathfrak{S}_{\bar{\alpha}}}{D X^{\bar{j}}} \psi_{+}^{\bar{j}} \chi_{-}^{\bar{\alpha}}-F_{\alpha \bar{\beta} i \bar{j}} \psi_{+}^{i} \psi_{+}^{\bar{j}} \chi_{-}^{\alpha} \chi_{-}^{\bar{\beta}},
$$

where

$$
F_{\alpha \bar{\beta} i \bar{j}}=-\partial_{\bar{j}} \partial_{i} h_{\alpha \bar{\beta}}+h^{\gamma \bar{\rho}}\left(\partial_{i} h_{\alpha \bar{\rho}}\right)\left(\partial_{\bar{j}} h_{\gamma \bar{\beta}}\right)
$$

and

$$
\frac{D \mathfrak{S}_{\alpha}}{D X^{j}}=\partial_{j} \mathfrak{S}_{\alpha}+h^{\beta \bar{\gamma}}\left(\partial_{j} h_{\alpha \bar{\gamma}}\right) \mathfrak{S}_{\beta} .
$$

\subsubsection{Relations with Hermitian Holomorphic Vector Bundle}

It turns out that we are describing a rank $r$ Hermitian holomorphic vector bundle $\mathbb{E} \rightarrow X$ over a Kähler manifold $X$ with Hermitian structure 
$h_{\alpha \bar{\beta}}$. Here we briefly summarize some properties of Hermitian holomorphic bundles [29]. Consider a rank $r$ complex vector bundle $\mathbb{E}$ over $X$. Let $\Omega^{p, q}(X, \mathbb{E})$ denote the space of $(p, q)$-forms over $X$ with values in $\mathbb{E}$. A connection (the covariant derivative) $d_{A}$ can be decomposed as

$$
d_{A}=\partial_{A}+\bar{\partial}_{A}: \Omega^{p, q}(X, \mathbb{E}) \rightarrow \Omega^{p+1, q}(X, E) \oplus \Omega^{p, q+1}(X, \mathbb{E}) .
$$

A connection $d_{A}$ endows $\mathbb{E}$ with a structure of a holomorphic vector bundle if the (0,2)-component $F^{0,2} \in \Omega^{2}(X, \operatorname{End}(\mathbb{E}))$ of its curvature $F$ vanishes, i.e., $\bar{\partial}_{A}^{2}=0$. A complex vector bundle $\mathbb{E}$ is Hermitian if it has a fixed Hermitian structure $h$ which is a $C^{\infty}$ field of positive definite Hermitian inner products in the fibers of $\mathbb{E}$. Given a local frame field $s_{U}=\left(s_{1}, \ldots, s_{r}\right)$ of $\mathbb{E}$ over an open subset $U \subset X$ we set $h_{\alpha \bar{\beta}}=h\left(s_{\alpha}, s_{\beta}\right)$ where $\alpha, \beta=1, \ldots, r$. Gluing them along different coordinate patches as usual we obtain $h_{\alpha \bar{\beta}}\left(z^{i}, z^{\bar{i}}\right)$. A connection $D$ in $(\mathbb{E}, h)$ is called an $h$-connection if $d(h(\xi, \eta))=h(D \xi, \eta)+h(\xi, D \eta)$ for $\xi, \eta \in \Omega^{0}(\mathbb{E})$. The theorem is that given a Hermitian structure $h$ in a holomorphic vector bundle $\mathbb{E}$, there is a unique $h$-connection $d_{A}$ called Hermitian connection such that $\bar{\partial}_{A}=\bar{\partial}$. Finally the curvature two-form of a Hermitian connection is of type $(1,1)$, thus $F^{2,0}$ also vanishes. The curvature two-form is given by the formula

$$
F_{\alpha \bar{\beta}}:=F_{\alpha \bar{\beta} \bar{j}} d z^{i} \wedge d z^{\bar{j}},
$$

where $F_{\alpha \bar{\beta} i \bar{j}}$ is defined as (2.78). We note that the Kähler metric $g_{i \bar{j}}$ on $X$ is a Hermitian structure of $T X$.

We saw that our model describes a rank $r$ Hermitian holomorphic vector bundle $\mathbb{E}$ with Hermitian structure $h_{\alpha \bar{\beta}}\left(z^{i}, z^{\bar{i}}\right)$. Now $\mathfrak{S}_{\alpha}$ can be identified with a holomorphic section of $\mathbb{E}$. In summary a $N_{c}=(2,0)$ model is associated with a Hermitian holomorphic vector bundle $(\mathbb{E}, h)$ over a Kähler manifold $X$ with holomorphic section. Associated with the base manifold $X$ we have holomorphic multiplets (2.72), as in the toy model. Associated with the fiber space we have Fermi multiplets (2.73).

\subsubsection{The Path Integrals}

Now we examine the path integral of our model in the various situations. 


\section{Turning Off the Holomorphic Section}

To begin with we consider the case that $\mathfrak{S}_{\alpha}=0$. The partition function $Z$ is defined by

$$
\begin{aligned}
Z=\int\left[\prod_{k, \bar{\kappa}=1}^{n}\left(d X^{k} d X^{\bar{k}} d \psi_{+}^{k} d \psi_{+}^{\bar{k}}\right) \prod_{\gamma, \bar{\gamma}=1}^{r}\left(d \chi_{-}^{\gamma} d \chi_{-}^{\bar{\gamma}}\right)\right] \\
\cdot \exp \left(F_{\alpha \bar{\beta} i \bar{j}} \psi_{+}^{i} \psi_{+}^{\bar{j}} \chi_{-}^{\alpha} \chi_{-}^{\bar{\beta}}\right) .
\end{aligned}
$$

The bosonic integral is an integral over $X$. As before the bosonic integral and integration over $\psi_{+}^{k}$ and $\psi_{+}^{\bar{k}}$ combine into the integration of differential forms on $X$ by replacing $F_{\alpha \bar{\beta} i \bar{j}} \psi_{+}^{i} \psi_{+}^{\bar{j}}$ with the curvature two-form $F_{\alpha \bar{\beta}}$ defined in (2.81). Thus we have

$$
Z=\int_{X} \prod_{\gamma, \bar{\gamma}=1}^{r}\left(d \chi_{-}^{\gamma} d \chi_{-}^{\bar{\gamma}}\right) \exp \left(F_{\alpha \bar{\beta}} \chi_{-}^{\alpha} \chi_{-}^{\bar{\beta}}\right) .
$$

The fermionic integral of $\chi^{\alpha}$ and $\chi^{\bar{\beta}}$ leads to the Paffian of the curvature two-form $F_{\alpha \bar{\beta}} \in \Omega^{1,1}(X, \operatorname{End}(\mathbb{E})$. We immediately see that the integrand is not a top form on $X$ unless $n=r$. For $n=r$ the partition function is the Euler character $\chi(\mathbb{E})$,

$$
Z=\int_{X} e(\mathbb{E})=\chi(\mathbb{E})
$$

otherwise, for $n \neq r$, the path integral vanishes. In the case $r<n$ we can insert a set of observables $\prod \widehat{\alpha}^{p_{\ell}, q_{\ell}}$ with the total ghost number $(n-r, n-r)$ and evaluate the correlation function

$$
\left\langle\prod_{\ell=1}^{m} \widehat{\alpha}^{p_{\ell}, q_{\ell}}\right\rangle=\int_{X} e(\mathbb{E}) \wedge \alpha^{p_{1}, q_{1}} \wedge \ldots \wedge \alpha^{p_{m}, q_{m}}
$$

The path integral always vanishes for $r>n$. We see that the vector bundle $\mathbb{E}$ after the parity change can be viewed as a bundle spanned by anti-ghosts $\chi_{-}^{\alpha}$ over $X$.

\section{Turning On the Holomorphic Section}

Now we turn on the holomorphic section $\mathfrak{S}_{\alpha}$ of $E \rightarrow X$. Applying the fixed point theorem of Witten we see that the path integral is 
localized to an $\boldsymbol{s}_{+}$and $\overline{\boldsymbol{s}}_{+}$invariant neighborhood of the vanishing locus $N$ of $\mathfrak{S}_{\alpha}\left(X^{i}\right)$ in $X$, where $\alpha=1, \ldots, r$ and $i=1, \ldots, n$. The condition $\mathfrak{S}_{\alpha}\left(X^{i}\right)=0$ implies $s_{+}(S)_{\alpha}=0$ in the $s_{+}$invariant neighborhood of $N$. We have

$$
\partial_{j} \mathfrak{S}_{\alpha} \psi_{+}^{j}=0
$$

We call a non-trivial solution above a zero-modes of $\psi_{+}$, which is a degree of freedom tangent to the vanishing locus $N$. We call a nontrivial solution of the similar equations

$$
\partial_{j} \mathfrak{S}_{\alpha} \chi_{-}^{\alpha}=0
$$

a zero-mode of $\chi_{-}$. For a generic choice of section $\mathfrak{S}_{\alpha}$ the equation $\mathfrak{S}_{\alpha}=0$ cuts out a $(n-r)$ complex dimensional subspace of $X$. Then the equation (2.86) implies that we have exactly $(n-r)$ zero-modes of $\psi_{+}$, while the equation (2.87) implies that we do not have any zeromodes of $\chi_{-}$, since $n \geq r$. Assume that the equations $\partial_{j} \mathfrak{S}_{\alpha}=0$, only for a fixed $\alpha$ have common roots for all $j=1, \ldots, n$. Then (2.86) for the fixed $\alpha$ do not impose any condition on the $\psi_{+}^{j}$ and we may have $(n-r+1)$ zero-modes of $\psi_{+}$. Similarly the equations $(2.87)$ do not impose any condition on the fixed component $\chi_{-}^{\alpha}$ and we may have one zero-mode of $\chi_{-}$. Thus we may draw two conclusions

1. For a generic choice of section we do not have any zero-modes of anti-ghosts. The vanishing locus $\mathfrak{S}^{-1}(0)$ of the section has the right complex $(n-r)$ dimensions and the zero-modes of $\psi_{+}$span the tangent space of $\mathfrak{S}^{-1}(0)$.

2. For a non-generic choice of section we may have anti-ghost zeromodes. The vanishing locus $\mathfrak{S}^{-1}(0)$ of the section have dimension higher than the right one. In any cases we have

$$
n-r=\#\left(\widetilde{\psi}_{+}\right)-\#\left(\widetilde{\chi}_{-}\right)
$$

where $\#(\widetilde{\text { fermi } i})$ denotes the number of fermionic zero-modes. We call the above the formal or virtual complex dimension of $\mathfrak{S}^{-1}(0)$. The space of anti-ghost zero-modes span a vector bundle $\mathbb{V}$ over $\mathfrak{S}^{-1}(0)$ called the anti-ghost bundle. The fiber dimension of $\mathbb{V}$ may jump when $\mathfrak{S}^{-1}(0)$ develops singularities.

We also see that our action functional $S^{\prime}$ (2.77) restricted to the $s_{+}$and $\bar{s}_{+}$invariant neighborhood $\mathcal{C}$ of the fixed point locus is 
given by

$$
\left.S^{\prime}\right|_{\mathcal{C}}=-F_{\alpha^{\prime} \bar{\beta}^{\prime} i^{\prime} \bar{j}^{\prime}} \widetilde{\psi}_{+}^{i^{\prime}} \widetilde{\psi}_{+}^{\bar{j}^{\prime}} \widetilde{\chi}_{-}^{\alpha^{\prime}} \widetilde{\chi}_{-}^{\bar{\beta}^{\prime}}
$$

where it is understood all the fermions $\left(\psi_{+}^{i}, \psi_{+}^{\bar{i}}, \chi_{-}^{\alpha}, \chi_{-}^{\bar{\alpha}}\right)$ are replaced by their zero-modes $\left(\widetilde{\psi_{+}^{i^{\prime}}}, \widetilde{\psi}_{+}^{i^{\prime}}, \widetilde{\chi}_{-}^{\alpha^{\prime}}, \chi_{-}^{\bar{\alpha}^{\prime}}\right)$ and the curvature above is the curvature of the anti-ghost bundle $\mathbb{V}$ over $\mathfrak{S}^{-1}(0)$.

Now we examine the path integral. For $n=r$ and with a generic section the vanishing locus $\mathfrak{S}^{-1}(0)$ is zero-dimensional and the path integral counts the number of zeros of the section. For $n=r$ and with a non-generic section the zeros of the section can be a positive dimensional submanifold $\mathfrak{S}^{-1}(0) \subset X$ of $X$. The path integral reduces to an integral over $\mathfrak{S}^{-1}(0)$ and over anti-ghost zero-modes. Note that the rank of the anti-ghost bundle $\mathbb{V}$ over $\mathfrak{S}^{-1}(0)$ is the same as the complex dimension of $\mathfrak{S}^{-1}(0)$. The path integral becomes $\chi(\mathbb{V})$

$$
Z=\int_{\mathfrak{S}^{-1}(0)} e(\mathbb{V})=\chi(\mathbb{V})
$$

which in turn can be identified with $\chi(\mathbb{E})$.

Now we consider the case $r<n$. The partition function still evaluates the Euler class $e(\mathbb{V})$ of the anti-ghost bundle $\mathbb{V}$ over $\mathfrak{S}^{-1}(0)$. Since, by the formula (2.88), the rank of $\mathbb{V}$ is smaller than the complex dimension of $\mathfrak{S}^{-1}(0)$. Thus the Euler class $e(\mathbb{V})$ is not a top form and the partition function vanishes. To get a non-trivial result we should insert a set of observables and evaluate the expectation value

$$
\left\langle\prod_{\ell=1}^{m} \widehat{\alpha}^{p_{\ell}, q_{\ell}}\right\rangle=\int_{\mathfrak{S}^{-1}(0)} e(\mathbb{V}) \wedge \alpha^{p_{1}, q_{1}} \wedge \ldots \wedge \alpha^{p_{m}, q_{m}}
$$

where

$$
\sum_{\ell=1}^{m} p_{\ell}=\sum_{\ell=1}^{m} q_{\ell}=n-r
$$

and otherwise the path integral vanishes. If there are no anti-ghost zeromodes we have $e(\mathbb{V})=1$ and the above correlation function reduces to the intersection number of homology cycles Poincaré dual to $\alpha^{p_{\ell}, q_{\ell}}$ in $\mathfrak{S}^{-1}(0)$. The selection rule above can be understood in more physical terms. The path integral measure contains a ghost number anomaly due to the fermionic zero-modes. The net ghost number violation of the 
path integral measure is $(n-r, n-r)$, which follows from the formula (2.88) and the ghost numbers of the fermions;

$$
\begin{array}{ll}
\psi_{+}^{i}:(1,0), & \chi_{-}^{\alpha}:(-1,0), \\
\psi_{+}^{\bar{i}}:(0,1), & \chi_{-}^{\bar{\alpha}}:(0,-1) .
\end{array}
$$

To cancel the ghost number anomaly we have to insert observables according to the selection rule (2.92) to soak up the fermion zero-modes in the path integral measure.

Specializing to $N_{c}=(2,2)$ Model

Finally we consider a special case of $N_{c}=(2,0)$ model which actually has $N_{c}=(2,2)$ supersymmetry. We have the following properties

1. For a generic choice of holomorphic potential $\mathcal{W}\left(X^{i}\right)$ we do not have any anti-ghost zero-modes. The critical set $V_{i}^{-1}(0)$ where $V_{i}=\partial_{i} \mathcal{W}\left(X^{j}\right)$ consists of a collection of non-degenerate points. The partition function is the number of such points.

2. For a non-generic $\mathcal{W}\left(X^{i}\right)$ we may have anti-ghost zero-modes. The critical set $V_{i}^{-1}(0)$ may be a higher dimensional subvariety of $X$. The net ghost number violation in the path integral measure is always zero. Thus the rank of the anti-ghost bundle $\mathbb{V}$ is exactly the same as the complex dimension of $V_{i}^{-1}(0)$. Thus the partition function is well-defined and computes the Euler characteristic $\chi(\mathbb{V})$ of $\mathbb{V}$. We can identify $\mathbb{V}$ with the tangent bundle of $V_{i}^{-1}(0)$. Thus the partition function is the Euler characteristic of $V_{i}^{-1}(0)$. This, in turn, can be identified with the Euler characteristic of $X$.

\section{Equivariant Cohomological Field The- ory}

In the previous chapter we developed standard models of cohomological field theories associated with a Kähler manifold $X$, tangent bundle $T X$ and Hermitian holomorphic vector bundle $\mathbb{E}$ over $X$. In this chapter 
we generalize those modes to the cases when there is a certain group $\mathcal{G}$ action. This generalization is relevant since most of field theory has a certain gauge symmetry. The models in the previous chapter are obviously empty if the target space $X$ is linear. On the other hand models in this chapter have rich structures both for linear ${ }^{9}$ and nonlinear target spaces. This also allows us to consider more general classes of target spaces like the space of a certain set of matrices, the space of a certain set of fields on a manifold, etc.

The central tool will be the notion of equivariant cohomology and symplectic quotients. The only practical difference between the models in the previous chapter and their equivariant generalizations are that the later models further localize the path integrals to the vanishing locus of $\mathcal{G}$-momentum map, modulo the $\mathcal{G}$ symmetry. If the $\mathcal{G}$ acts freely on such locus we recover the standard models in the previous chapters now associated with the symplectic quotients. The momentum map is a generalization of the familiar angular momentum associated with a group of rotations in the classical mechanics.

\subsection{Equivariant Toy Model}

We return to our toy model in Sect. 2.1.1, where we considered a $n$ dimensional Kähler manifold $(X, \varpi)$ with Kähler form $\varpi$ as the target space. Now we assume that there is a group $\mathcal{G}$ action

$$
\mathcal{G} \times X \rightarrow X
$$

preserving the complex and Kähler structures. We consider the toy model with the action functional $S$ in (2.4). The action functional is invariant under $\mathcal{G}$ thus the path integral is degenerated. We want to

\footnotetext{
${ }^{9}$ The relation between the previous section and the present section is best compared with that of non-linear sigma-models and linear gauged sigma models in two-dimensions.
} 
remove the gauge degree of freedom as follows (compare with (2.8))

$$
\begin{aligned}
Z & =\frac{1}{\operatorname{vol}(\mathcal{G})} \int_{X}[\mathcal{D} X \mathcal{D} \bar{X} \mathcal{D} \psi \mathcal{D} \bar{\psi}] e^{-S} \\
& =\frac{1}{\#(\mathcal{G})} \int_{X / \mathcal{G}}[\mathcal{D} X \mathcal{D} \bar{X} \mathcal{D} \psi \mathcal{D} \bar{\psi}]^{\prime} e^{-S} \\
& =\frac{1}{\#(\mathcal{G})} \int_{X}[\mathcal{D} X \mathcal{D} \bar{X} \mathcal{D} \psi \mathcal{D} \bar{\psi} \mathcal{D}(\text { ghosts })] e^{-S-S_{g f}-S_{g h}}
\end{aligned}
$$

where $\#(\mathcal{G})$ denotes the number of central elements of $\mathcal{G}, S_{g f}$ and $S_{g h}$ denote the gauge fixing and ghost terms. The above procedure is the well-known Faddeev-Popov-BRST quantization on which I do not want to review here. ${ }^{10}$

A general problem with the path integral above is that the quotient space $X / \mathcal{G}$ rarely has good topology and geometry. This means that it is difficult make sense out of our (even for finite dimensional) path integral. Furthermore the geometrical meaning of the $s$ and $\bar{s}$ supercharges on the quotient space is not quite obvious. This problem can be avoided by considering equivariant cohomology. For general references see [24], [30], [31].

\subsubsection{Extending Our Toy Model}

A nice route to introduce the equivariant cohomology is a simple generalization of our toy model in Sect. 2.1.1. Now we assume that there is a group $\mathcal{G}$ action $\mathcal{G} \times X \rightarrow X$ on our target space $X$ preserving the complex and Kähler structures. Our goal is to extend our target space and supercharges $s$ and $\bar{s}$ by introducing extra fields such that

1. If $\mathcal{G}$ acts freely on $X$ the degrees of freedom due to the extra fields disappear,

2. the supercharges become $\partial$ and $\bar{\partial}$ operators, after the parity change, on the $\mathcal{G}$-invariant subspace.

\footnotetext{
${ }^{10}$ I only want to remark that it involves the Lie algebra cohomology with the parity change.
} 
To implant the above idea we need the notion of Lie derivative. Consider a manifold with $\mathcal{G}$ action. Let $\operatorname{Lie}(\mathcal{G})$ be the Lie algebra of $\mathcal{G}$. We will always assume that we have a bi-invariant inner product $\langle$, on $\operatorname{Lie}(\mathcal{G})$ such that we can identify $\operatorname{Lie}(\mathcal{G})$ with its dual $\operatorname{Lie}(\mathcal{G})^{*}$. Let $X^{I}$ be the local coordinate fields on $X$. The $\mathcal{G}$ action induces a vector $V_{a}^{I} T^{a}$ such that an infinitesimal $\mathcal{G}$ action is represent by

$$
X^{I} \rightarrow X^{I}+\varepsilon^{a} V_{a}^{I} .
$$

We denote by $j_{a}$ the interior derivative with respect to the vector $V_{a}$, i.e.,

$$
\begin{gathered}
j_{a}: \Omega^{r}(X) \rightarrow \Omega^{r-1}(X), \\
\left(j_{a} \alpha\right)_{I_{2} I_{3} \cdots I_{r}}=r V^{I_{1}} \alpha_{I_{1} I_{2} \cdots I_{r}} .
\end{gathered}
$$

Let $\mathcal{L}_{a}$ be the Lie derivative with respect to the vector field $V_{a}$;

$$
\mathcal{L}_{a}=d j_{a}+j_{a} d
$$

Then the infinitesimal $\mathcal{G}$ action on $\alpha \in \Omega^{*}(X)$ is given by $\alpha \rightarrow \alpha+$ $\varepsilon^{a} \mathcal{L}_{a} \alpha$. Thus a differential form $\alpha$ is $\mathcal{G}$-invariant if $\mathcal{L}_{a} \alpha=0$. We note an obvious relation $\varepsilon^{a} \mathcal{L}_{a} X^{I}=\varepsilon^{a} V_{a}^{I}$.

Now we extend our target space $X$ by introducing a $\operatorname{Lie}(\mathcal{G})$-valued scalar $\phi=\phi^{a} T_{a}$ and modify the commutation relation (2.2) as ${ }^{11}$

$$
s^{2}=0, \quad\{s, \bar{s}\}=-i \phi^{a} \mathcal{L}_{a}, \quad \bar{s}^{2}=0 .
$$

Thus $\{s, \bar{s}\}=0$ on the $\mathcal{G}$-invariant subspace of $X$ and the supercharges are related with the $\partial$ and $\bar{\partial}$ operators on the invariant subspace as in the case of our previous toy model. The ghost numbers of $\phi$ should be assigned $(1,1)$ to match the ghost numbers in the anti-commutation relations above. The above defines $\mathcal{G}$-equivariant Dolbeault cohomology [32], [33].

By the new anti-commutation relations (3.6) the supersymmetry transformation laws (2.4) should be modified as follows

$$
\begin{array}{lll}
s X^{i}=i \psi^{i}, & s \psi^{i}=0, & \\
\bar{s} X^{i}=0, & \bar{s} \psi^{i}=-\phi^{a} \mathcal{L}_{a} X^{i}, & s \phi=0, \\
s X^{\bar{i}}=0, & s \psi^{\bar{i}}=-\phi^{a} \mathcal{L}_{a} X^{\bar{i}}, & \bar{s} \phi=0, \\
\bar{s} X^{\bar{i}}=i \psi^{\bar{i}}, & \overline{\boldsymbol{s}} \psi^{\bar{i}}=0 . &
\end{array}
$$

\footnotetext{
${ }^{11}$ where $\phi^{a}$ is $\varepsilon^{a}$ in (3.3) incarnated as a field.
} 
where we obtained the conditions $s \phi=\bar{s} \phi=0$ by demanding the algebra to be closed. Assume that we have a model with an action functional which is invariant under the supersymmetries generated by the above new supercharges. Then we can apply the fixed point theorem of Witten and we have the following fixed point equation, deduced from the above

$$
\phi^{a} \mathcal{L}_{a} X^{I}=0 .
$$

This equation tells us that $\phi^{a}=0$ if $\mathcal{G}$ act freely on $X$ while $\phi^{a}$ can be non-zero on a fixed point of the $\mathcal{G}$ action. Thus we achieved our initial two goals.

Now we consider a supersymmetric action functional $S$. Compare with the non-equivariant case in Sect. 2.1.1, an action functional should be invariant under $\mathcal{G}$ in addition to $s S=\bar{s} S=0$. These conditions imply that one can also apply the Poincaré lemma since the new supercharges are also nilpotent if they are acting on $\mathcal{G}$ invariant quantities. Thus $S$ can be written by the same form as the previous toy model

$$
S=i s \bar{s} \mathcal{K}\left(X^{i}, X^{\bar{i}}\right)
$$

where $\mathcal{K}$ should be $\mathcal{G}$ invariant. ${ }^{12}$ Applying the transformation laws (3.7) we have

$$
S=-i\langle\phi, \mu\rangle-i g_{i \bar{j}} \psi^{i} \psi^{\bar{j}}
$$

where

$$
\mu_{a}=i \frac{\partial \mathcal{K}}{\partial X^{\bar{i}}}\left(\mathcal{L}_{a} X^{\bar{i}}\right)
$$

Later we shall see that $\mu=\mu_{a} T^{a}$ is the equivariant $\mathcal{G}$ momentum map on $X$. Maintaining all the supersymmetry we consider the following more general action functional $S(\zeta)$

$$
\begin{aligned}
S(\zeta) & =i s \bar{s} \mathcal{K}+i \phi^{a} \zeta_{a} \\
& =-i\langle\phi, \mu-\zeta\rangle-i g_{i \bar{j}} \psi^{i} \psi^{\bar{j}}
\end{aligned}
$$

where $\zeta$ belongs to the center of $\mathcal{G}$. We call the additional term a FI coupling.

\footnotetext{
${ }^{12}$ Actually $\mathcal{K}$ only needs to satisfy a weaker condition that it should invariant under gauge transformations connected to the identity.
} 
Now we consider the partition function for the new action. We have

$$
\begin{aligned}
Z(\zeta) & =\frac{1}{\operatorname{vol}(\mathcal{G})} \int[\mathcal{D} \phi \mathcal{D} X \mathcal{D} \bar{X} \mathcal{D} \psi \mathcal{D} \bar{\psi}] e^{-S(\zeta)} \\
& =\frac{1}{\operatorname{vol}(\mathcal{G})} \int_{X} \delta(\mu-\zeta) \prod_{k, \bar{k}} d X^{\kappa} d X^{\bar{k}} d \psi^{k} d \psi^{\bar{k}} \cdot e^{-i g_{i \bar{j}} \psi^{i} \psi^{\bar{j}}} \\
& =\frac{1}{\# \mathcal{G}} \int_{\mu^{-1}(\zeta) / \mathcal{G}} \frac{\widetilde{\varpi}^{r}}{r !} \\
& =\frac{1}{\# \mathcal{G}} \operatorname{vol}\left(\mathcal{N}_{\zeta}\right)
\end{aligned}
$$

where

$$
\mathcal{N}_{\zeta}=\mu^{-1}(\zeta) / \mathcal{G}
$$

In the above we assumed that $\mathcal{G}$ acts freely on the locus $\mu^{-1}(\zeta) \subset X$. Thus we could simply integrate $\phi$ out, which gives rise to the delta function supported on $\mu^{-1}(\zeta)$. Then the quotient space $\mathcal{N}_{\zeta}$ is smooth. Our action functional $S_{\zeta}$ reduces to the Kähler form on the subspace $\mu^{-1}(\zeta)$. Since it is $\mathcal{G}$ invariant it becomes, after the parity change, the Kähler from $\widetilde{\varpi}$ on the quotient space $\mathcal{N}_{\zeta}$. What we showed is the symplectic reduction theorem of Marsden and Weinstein [34].

We call our extended toy model the equivariant toy model. We note that the equivariant toy model makes perfect sense even if we start from a flat Kähler manifold $X$ as our initial target space. We call the space $\mathcal{N}_{\zeta}$ the effective target space, which can be a very complicated non-linear space even if our initial target space $X$ is flat.

Before examining further properties of our model, we turn to a review of the equivariant cohomology and momentum map. We refer for details on the equivariant cohomology and relation with momentum maps to a beautiful exposition of Atiyah and Bott [30]. The idea is to replace $X$ by a bigger space $X \times E \mathcal{G}$ such that the extended space has a nice quotient

$$
X_{\mathcal{G}}=\left(X \times_{\mathcal{G}} E \mathcal{G}\right)
$$

which is equivalent to the original quotient $M / \mathcal{G}$ when it has a nice quotient. ${ }^{13}$ The $\mathcal{G}$-equivariant cohomology $H_{\mathcal{G}}^{*}(X)$ of $X$ is defined as the ordinary cohomology $H^{*}\left(X_{\mathcal{G}}\right)$ of $X_{\mathcal{G}}$. For instance the $\mathcal{G}$-equivariant

\footnotetext{
${ }^{13}$ The additional space $E \mathcal{G}$ is a fixed universal $\mathcal{G}$-bundle over the classifying space $B \mathcal{G}$. The homotopy quotient $X_{\mathcal{G}}$ forms a fiber bundle $\pi: X_{\mathcal{G}} \rightarrow B \mathcal{G}$ with fiber $X$.
} 
cohomology of $M$ is the ordinary cohomology of $X / \mathcal{G}$ if $\mathcal{G}$ acts freely on $X .^{14}$

We will briefly review a convenient model of equivariant cohomology due to Cartan, of which variants will be used in this thesis. A crucial reference on the Cartan model for us is Witten's paper [35]. The path integral of the equivariant toy model reproduces a Kähler version of Witten's non-Abelian equivariant integration formula.

\subsubsection{Equivariant Cohomology and Momentum Map}

Consider a manifold $X$ with $\mathcal{G}$ action. Let $\operatorname{Lie}(\mathcal{G})$ be the Lie algebra of $\mathcal{G}$. We will always assume that we have a bi-invariant inner product $\langle$,$\rangle on \operatorname{Lie}(\mathcal{G})$ such that we can identify $\operatorname{Lie}(\mathcal{G})$ with its dual $\operatorname{Lie}(\mathcal{G})^{*}$.

Let $\operatorname{Fun}^{*}(\operatorname{Lie}(\mathcal{G}))$ denote the algebra of polynomial functions on $\operatorname{Lie}(\mathcal{G})$ so that an $m^{\text {th }}$ order homogeneous polynomial is considered to be of degree $2 m$. The equivariant differential forms $\Omega_{\mathcal{G}}^{*}(X)$ on $X$ are represented by

$$
\Omega_{\mathcal{G}}^{*}(X):=\left(\Omega^{*}(X) \otimes F u n^{*}(\operatorname{Lie}(\mathcal{G}))\right)^{\mathcal{G}},
$$

where ${ }^{\mathcal{G}}$ denote the $\mathcal{G}$-invariant part. The degree of such a form is the sum of degrees of $\Omega^{*}(X)$ and $F u n^{*}(\operatorname{Lie}(G))$. One endows $\Omega_{\mathcal{G}}^{*}(X)$ with the equivariant differential operator $d_{\mathcal{G}}$

$$
d_{\mathcal{G}}=d-i \phi^{a} j_{a}, \quad d_{\mathcal{G}}^{2}=-i \phi^{a} \mathcal{L}_{a},
$$

where $j_{a}^{2}=0$ and $\phi=\phi^{a} T^{a} \in \operatorname{Lie}(\mathcal{G})$. That is, $d_{\mathcal{G}}^{2}=0$ modulo an infinitesimal gauge transformation generated by $\phi^{a}$. Thus on the space $\Omega_{\mathcal{G}}^{*}(X)$ we have $\mathrm{w}^{15}$

$$
\widehat{d}_{\mathcal{G}}^{2}=0
$$

Then we have the following diagram

$$
\begin{array}{cccc}
E \mathcal{G} & \leftarrow & E \mathcal{G} \times X & \rightarrow X \\
\downarrow & & \downarrow & \downarrow \\
B \mathcal{G} & \leftarrow & E \mathcal{G} \times \mathcal{G} X & \rightarrow X / \mathcal{G} .
\end{array}
$$

${ }^{14}$ Note, however, $\mathcal{G}$-equivariant cohomology of a point is $H^{*}(B \mathcal{G})$ which is highly non-trivial.

${ }^{15} \mathrm{An}$ element in $\Omega_{\mathcal{G}}^{*}(X)$ is annihilated by $L_{a}=\mathcal{L}_{a}+f_{a b}{ }^{c} \phi^{b} \frac{\partial}{\partial \phi^{c}}$, where $f_{a b}{ }^{c}=$ $-f_{b a}{ }^{c}$ are the structure constants of $\mathcal{G}$. Then, it is also annihilated by $\phi^{a} \mathcal{L}_{a}$ since $\phi^{a} L_{a}=\phi^{a} \mathcal{L}_{a}$. 
The $\mathcal{G}$-equivariant de Rham cohomology on $X$ is the cohomology of the complex $\left(\Omega_{\mathcal{G}}^{*}(X), d_{\mathcal{G}}\right)$. The equivariant cohomology of $X$ is the ordinary cohomology of the quotient space if the group acts freely, otherwise it is something else. For example $H_{\mathcal{G}}^{*}(p t)$ is $\operatorname{Fun}^{*}(\operatorname{Lie}(\mathcal{G}))$.

\section{The Symplectic Case}

Now we consider a symplectic manifold $X$ with symplectic form $\varpi$. Assume that we have a $\mathcal{G}$ action on $X$. Under an infinitesimal $\mathcal{G}$ action $X^{I} \rightarrow X^{I}+\varepsilon^{a} V_{a}^{I}$ the symplectic form transforms as $\varpi \rightarrow \varpi+\varepsilon^{a} \mathcal{L}_{a} \varpi$. Thus we have a vector field $V_{a}^{I}$ which is an infinitesimal symplectic transformation whenever $\mathcal{L}_{a} \varpi=0$. Since $d \varpi=0$ we have $d\left(j_{a} \varpi\right)=0$, thus at least locally we can write

$$
j_{a} \varpi=d \mu_{a}
$$

The $\mu=\mu_{a} T^{a}: X \rightarrow \operatorname{Lie}(\mathcal{G})^{*}$ is called the $\mathcal{G}$-momentum map. ${ }^{16}$ The obstruction for global existence of $\mu_{a}$ is $H^{1}(X)$. The momentum map is a generalization of the familiar classical mechanical notion that $X$ is a classical phase space and $\mathcal{G}$ is a group of rotation and $\mu$ is the angular momentum. The momentum map is equivariant if $\mu(g(x))=$ $(a d g)^{*}(\mu(x))$. Then $\mathcal{G}$ preserve the subspace $\mu^{-1}(\zeta)$ when $\zeta$ is a centeral element. Then the reduced phase space or the symplectic quotient is defined by

$$
\mathcal{N}_{\zeta}=\left(X \cap \mu^{-1}(\zeta)\right) / \mathcal{G}
$$

The quotient space is a smooth symplectic manifold if $\zeta$ is a regular value. The symplectic form $\widetilde{\varpi}$ on $N_{\zeta}$ is obtained from $\varpi$ by restriction and reduction [34].

The equivariant cohomology and the momentum map are closely related [30]. Note that the symplectic form $\varpi$ is not equivariantly closed, $d_{\mathcal{G}} \varpi \neq 0$. We have a unique form, due to the degree, of equivariant extension $\varpi_{\mathcal{G}}$ of $\varpi$

$$
\varpi_{\mathcal{G}}=\varpi+i(\phi, \mu)
$$

The condition $d_{\mathcal{G}} \varpi_{\mathcal{G}}=0$ reduces to using $d_{\mathcal{G}} \phi=0$

$$
\langle\phi, d \mu-j \varpi\rangle=0 .
$$

\footnotetext{
${ }^{16}$ Note that we identified $\operatorname{Lie}(\mathcal{G})$ with its dual $\operatorname{Lie}(\mathcal{G})^{*}$.
} 
Thus $\varpi_{\mathcal{G}}$ is equivariantly closed iff $\mu$ is the momentum map (3.18). Note that $\varpi_{\mathcal{G}}$ is $\mathcal{G}$ invariant, $L_{a} \varpi_{\mathcal{G}}=0$, iff the momentum map $\mu$ is equivariant.

\section{The Kähler Case}

Now we specialize to the case that $X$ is a Kähler manifold with Kähler form $\varpi$ and with $\mathcal{G}$ action, which preserve the complex structure and the Kähler form. The vector field $V^{I}$ induced by the $\mathcal{G}$ action is decomposed into $V^{I}=V^{i}+V^{\bar{i}}$. Thus one can introduce interior derivatives $\iota_{a}$ and $\bar{\iota}_{a}$ by contracting with $V_{a}^{i}$ and $V_{a}^{\bar{i}}$, respectively, such that $j_{a}=\iota_{a}+\bar{\iota}_{a}$;

$$
\begin{aligned}
& \iota_{a}: \Omega^{p, q}(X) \rightarrow \Omega^{p-1, q}(X), \\
& \bar{\iota}_{a}: \Omega^{p, q}(X) \rightarrow \Omega^{p, q-1}(X) .
\end{aligned}
$$

From the relation $j_{a}^{2}=0$ we have

$$
\iota_{a}^{2}=0, \quad\left\{\iota_{a}, \bar{\iota}_{a}\right\}=0, \quad \bar{\iota}_{a}^{2}=0 .
$$

It follows that

$$
\mathcal{L}_{a}=\partial \iota_{a}+\iota_{a} \partial+\bar{\partial} \bar{\iota}_{a}+\bar{\iota}_{a} \bar{\partial}
$$

We also decompose $F_{u n}^{*}(\operatorname{Lie}(\mathcal{G}))$ such that an $m^{\text {th }}$ order homogeneous polynomial in Fun $(\operatorname{Lie}(\mathcal{G}))$ is considered to be of degree $(m, m)$. Then equivariant differential forms $\Omega_{\mathcal{G}}^{*, *}(X)$ on $X$ are represented by

$$
\Omega_{\mathcal{G}}^{*, *}(X):=\left(\Omega^{*, *}(X) \otimes \operatorname{Fun}^{*}(\operatorname{Lie}(\mathcal{G}))\right)^{\mathcal{G}}
$$

Similarly we decompose $d_{G}$ into

$$
d_{\mathcal{G}}=\partial_{G}+\bar{\partial}_{G}: \Omega_{\mathcal{G}}^{0}(M)=\Omega_{\mathcal{G}}^{1,0}(M) \oplus \Omega_{\mathcal{G}}^{0,1}(M) .
$$

where

$$
\begin{aligned}
& \partial_{\mathcal{G}}=\partial-i \phi^{a} \bar{\iota}_{a}, \\
& \bar{\partial}_{\mathcal{G}}=\bar{\partial}-i \phi^{a} \iota_{a} .
\end{aligned}
$$

Remark that $\phi$ is assigned to degree $(1,1)$. The anti-commutation relations between $\partial_{\mathcal{G}}$ and $\bar{\partial}_{\mathcal{G}}$ are

$$
\partial_{\mathcal{G}}^{2}=0, \quad\left\{\partial_{\mathcal{G}}, \bar{\partial}_{\mathcal{G}}\right\}=-i \phi^{a} \mathcal{L}_{a}, \quad \bar{\partial}_{\mathcal{G}}^{2}=0
$$


This defines equivariant Dolbeault cohomology on a Kähler manifold. Comparing with the anti-commutation relations (3.6) we can identify our supercharges $s$ and $\bar{s}$ with $\partial_{\mathcal{G}}$ and $\bar{\partial}_{\mathcal{G}}$ after the parity change (2.16). Thus

$$
\begin{aligned}
& s=i \psi^{i} \frac{\partial}{\partial X^{i}}-\phi^{a} V_{a}^{\bar{i}} \frac{\partial}{\partial \psi^{\bar{i}}}, \\
& \bar{s}=i \psi^{\bar{i}} \frac{\partial}{\partial X^{\bar{i}}}-\phi^{a} V_{a}^{i} \frac{\partial}{\partial \psi^{i}} .
\end{aligned}
$$

Now we examine the relation between the momentum map and equivariant Dolbeault cohomology. For the Kähler case the relation (3.18) becomes, by matching form degrees

$$
\iota_{a} \varpi=\bar{\partial} \mu_{a} .
$$

Since the Kähler form $\varpi$ can be written locally in terms of a Kähler potential $f$,

$$
\varpi=i \partial \bar{\partial} f
$$

we have

$$
i \iota_{a}(\partial \bar{\partial} f)=\bar{\partial} \mu_{a}
$$

Using the relations $\left\{\iota_{a}, \bar{\partial}\right\}=\{\partial, \bar{\partial}\}=0$ we deduce that

$$
\mu_{a}=i \iota_{a}(\partial f)
$$

up to a constant. Combining all together we find an important identity

$$
i \partial_{\mathcal{G}} \bar{\partial}_{\mathcal{G}} f=\varpi+i\langle\phi, \mu\rangle,
$$

which we obtained earlier in (3.9) and (3.10). Thus minus the action functional, $-S$, is a $\mathcal{G}$-equivariant Kähler form after the parity change. Note that the momentum map derived above is equivariant if the Kähler potential is $\mathcal{G}$ invariant. Thus we showed all the assertions made in Sect. 3.1.1.

\subsubsection{The Path Integrals and Non-Abelian Localization The- orem}

We now return to the equivariant toy model. We return to the partition function $Z$ (3.13) and ask what will happen as we vary the FI term $\zeta$. 
We have a classical theorem; the image of a proper momentum map of a compact group is a convex polytope divided by walls [36], [37], [38]. As we vary $\zeta$ the symplectic quotient $\mathcal{N}_{\zeta}$ may undergo birational transformations if the path of $\zeta$ crosses a wall, otherwise diffeomorphic. For the non-proper case the symplectic quotient does not exist. This does not imply that the partition function is empty. Recall that space of all bosonic fields is a copy of $X$ and the space of all $\phi$. The correct picture is that the path integral is localized to $\mathcal{N}_{\zeta} \subset X / \mathcal{G}$ for regular values of $\zeta$. The full equation for the localization is

$$
\begin{aligned}
\mu-\zeta & =0 \\
\phi^{a} \mathcal{L}_{a}\left(X^{i}\right) & =0 .
\end{aligned}
$$

We call the non-trivial solutions $\phi_{0}$ of above equations the zero-modes of $\phi$. It is clear that we have zero-modes of $\phi$ whenever the $\mathcal{G}$ action has fixed points in $\mu^{-1}(\zeta)$, thus when $\zeta$ lies on a non-regular value $\zeta_{0}$. Clearly the path integral degenerates at such a value since the path integral measure contains zero-modes of $\phi$. Let $\zeta_{+}<\zeta_{0}<\zeta_{-}$we have

$$
Z\left(\zeta_{+}\right) \neq Z\left(\zeta_{-}\right)
$$

due to topology change. At $\zeta_{0}$ the partition function should be singular.

It is clear how to resolve the singularity of the path integral. We have to regularize. We consider a more general action functional $S(\zeta, \varepsilon)$,

$$
\begin{aligned}
S(\zeta, \varepsilon) & =S(\zeta)+\frac{\varepsilon}{2}\langle\phi, \phi\rangle \\
& =-i\langle\phi, \mu-\zeta\rangle+\frac{\varepsilon}{2}(\phi, \phi)-i g_{i \bar{j}} \psi^{i} \psi^{\bar{j}}
\end{aligned}
$$

Note that the additional term is invariant under $\mathcal{G}$ as well as all the supersymmetry. The additional $\varepsilon$ dependent term changes the fixed point equations of the supersymmetry since the $\phi$ equation of motion is now

$$
i(\mu-\zeta)=\varepsilon \phi
$$

Consequently, for $\varepsilon \neq 0$, the path integral is localized to the locus of the following equations

$$
\left(\frac{\partial \mu_{a}}{\partial X}\right)\left(\mu^{a}-\zeta^{a}\right)=0
$$


Now we also have contributions from higher critical points. Thus, we have two branches; (i) $\mu^{a}-\zeta^{\alpha}=0$, (ii) $\frac{\partial}{\partial X} \mu=0$. Clearly the quotient $\mathcal{N}_{\zeta}$ space develops singularities when branches (i) and (ii) intersect. In such a case the integrand of path integral contains the Gaussian measure

$$
e^{-\varepsilon \sum_{\ell}\left|\phi_{0, \ell}\right|^{2}}
$$

for the space of zero-modes $\phi_{0, \ell}$ of $\phi$. Thus the path integral is nonsingular. Consequently the politically correct version of the model is defined by the action functional $S(\zeta, \varepsilon)$.

Now we consider the correlation functions. A supersymmetric observable should be $\mathcal{G}$-invariant as well as invariant under $s$ and $\bar{s}$. Such an observable should be constructed from an equivariantly closed differential form. An equivariant differential form $\mathcal{O}^{p, q}$ of total degree $(p, q)$ can be expanded as

$$
\mathcal{O}^{p, q}=\alpha_{0}^{p, q}+\phi^{a} \alpha_{a}^{p-1, q-1}+\phi^{a} \phi^{b} \alpha_{a b}^{p-2, q-2}+\cdots,
$$

where $\alpha_{*}^{p, q} \in \Omega^{p, q}(X)$. Let $\widehat{\mathcal{O}}^{p, q}$ be the parity change of $\mathcal{O}^{p, q}$, thus carrying ghost number $(p, q)$. We have

$$
\bar{s}^{p, q}={\widehat{\bar{\partial}_{\mathcal{G}} \mathcal{O}}}^{p, q}
$$

Thus $\widehat{\mathcal{O}}^{p, q}$ is an $\bar{s}$-invariant observable if $\mathcal{O}^{p, q}$ is an $\bar{\partial}_{\mathcal{G}^{-}}$-losed equivariant differential form.

The correlation function of observables or the expectation value is defined by

$$
\left\langle\prod_{m=1}^{r} \widehat{\mathcal{O}}^{p_{m}, q_{m}}\right\rangle=\int[\mathcal{D} \phi \mathcal{D} X \mathcal{D} \bar{X} \mathcal{D} \psi \mathcal{D} \bar{\psi}] \prod_{m=1}^{r} \widehat{\mathcal{O}}^{p_{m}, q_{m}} \cdot e^{-S} .
$$

For the present model one can show that

$$
\begin{aligned}
& \left\langle\prod_{m=1}^{r} \widehat{\mathcal{O}}^{p_{m}, q_{m}}\right\rangle=\frac{1}{\operatorname{vol}(\mathcal{G})} \int_{\mathcal{G}} \frac{d \phi_{1} d \phi_{2} \ldots d \phi_{s}}{(2 \pi)^{s}} \\
& \times \int_{X} \mathcal{O}^{p_{1}, q_{2}} \wedge \ldots \wedge \mathcal{O}^{p_{r}, q_{r}} \cdot \exp \left(\varpi+i(\phi, \mu-\zeta)-\frac{\varepsilon}{2}(\phi, \phi)\right),
\end{aligned}
$$

where $s=\operatorname{dim}(\mathcal{G})$. Applying the fixed point theorem for the global supersymmetry we see that the above integral can be written as a sum 
of contribution of the critical points (3.39) of $I=\langle\mu, \mu\rangle$. This is the non-Abelian localization theorem of Witten [35], generalizing the more familiar abelian Duistermaat-Heckman (DH) integration formula [36]. In the end our equivariant toy model turns out to be very non-trivial.

\subsection{The Equivariant $N_{c}=(2,2)$ Model}

In this section we develop the equivariant generalization of the $N_{c}=$ $(2,2)$ model in Sect. 2.2. We assume the same group $\mathcal{G}$ acting on $X$ as in the previous section. This naturally extend to the tangent space $T X$. Recall that the partition function of our toy model is the symplectic volume of the target space, while the partition function of the equivariant toy model is the symplectic volume of the symplectic quotient $\mathcal{N}_{\zeta}$, for generic values of $\zeta$, of $X$ by $\mathcal{G}$. Similarly, the partition function the equivariant version of $N_{c}=(2,2)$ model, without holomorphic potential $\mathcal{W}$, will be the Euler characteristic $\chi\left(T \mathcal{N}_{\zeta}\right)$ of the symplectic quotient $\mathcal{N}_{\zeta}$, for generic value of $\zeta$, of $X$ by $\mathcal{G}$. After turning on $\mathcal{W}$, the path integral reduces to the symplectic quotient $\mathcal{M}_{\zeta}$ of the critical subset $X_{\text {crit }} \subset X$ of the potential $\mathcal{W}$ by $\mathcal{G}$.

We consider the same "type" of supercharges carrying the same ghost numbers $(p, q)$;

$$
\begin{array}{ll}
\boldsymbol{s}_{+}:(+1,0), & \overline{\boldsymbol{s}}_{+}:(0,+1), \\
\boldsymbol{s}_{-}:(-1,0), & \overline{\boldsymbol{s}}_{-}:(0,-1) .
\end{array}
$$

Now we postulate the supercharges to satisfy the following anti-commutation relations

$$
\begin{array}{lll}
\left\{\boldsymbol{s}_{+}, \boldsymbol{s}_{+}\right\}=0, & \left\{\boldsymbol{s}_{+}, \overline{\boldsymbol{s}}_{+}\right\}=-i \phi_{++}^{a} \mathcal{L}_{a}, & \left\{\overline{\boldsymbol{s}}_{+}, \overline{\boldsymbol{s}}_{+}\right\}=0 \\
\left\{\boldsymbol{s}_{+}, \boldsymbol{s}_{-}\right\}=0, & \left\{\boldsymbol{s}_{+}, \overline{\boldsymbol{s}}_{-}\right\}=-i \sigma^{a} \mathcal{L}_{a}, \quad\left\{\overline{\boldsymbol{s}}_{+}, \overline{\boldsymbol{s}}_{-}\right\}=0 \\
\left\{\boldsymbol{s}_{-}, \boldsymbol{s}_{-}\right\}=0, & \left\{\overline{\boldsymbol{s}}_{+}, \boldsymbol{s}_{-}\right\}=-i \bar{\sigma}^{a} \mathcal{L}_{a}, \quad\left\{\bar{s}_{-}, \overline{\boldsymbol{s}}_{-}\right\}=0
\end{array}
$$

which are equivariant generalizations of the commutation relations $(2.26)$ and $(2.27)$ for the $N_{c}=(2,2)$ model. For the $\mathcal{G}$-invariant subspace the equivariant supercharges are the same as the non-equivariant ones. Here, in total, we introduced four bosonic fields $\phi_{ \pm \pm}, \sigma$ and $\bar{\sigma}$ 
taking values in $\operatorname{Lie}(\mathcal{G})$. They carry the following ghost numbers

$$
\begin{array}{ll}
\phi_{++}:(+1,+1), & \sigma:(+1,-1), \\
\phi_{--}:(-1,-1), & \bar{\sigma}:(-1,+1) .
\end{array}
$$

The anti-commutation relations above define balanced $\mathcal{G}$-equivariant Dolbeault cohomology [39]. This is the Kähler version of the balanced equivariant cohomology [23]. ${ }^{17}$

We should remark that the above algebra can be obtained by dimensional reduction of the $N=1$ supersymmetry algebra of fourdimensional super-Yang-Mill theory and, equivalently, the algebra of $N_{w s}=(2,2)$ super-Yang-Mills theory in two-dimensions. Thus we may introduce other quantum numbers, as in two-dimensions, the left and right $U(1) \mathcal{R}$-charges $\left(J_{L}, J_{R}\right)$ as follows

$$
\begin{array}{ll}
s_{+}:(+1,0), & \bar{s}_{+}:(-1,0), \\
s_{-}:(0,+1), & \bar{s}_{-}:(0,-1) .
\end{array}
$$

The analogy with the two-dimensional $N_{w s}=(2,2)$ space-time supersymmetric gauge theory, equivalently the linear gauged sigma-model [18], [19] will be very useful. Indeed it is a trivial step to obtain a $N_{w s}=(2,2)$ model, and vice versa, just by replacing $\phi_{ \pm \pm}^{a} \mathcal{L}_{a}$ by the left and right moving covariant derivatives $D_{ \pm \pm}$everywhere. Then the indices \pm are identified with the left and right spinor indices in two-dimensions. For example requiring the ghost number symmetry is equivalent to requiring the two-dimensional Lorentz symmetry.

\subsubsection{The Basic Structure}

Now we examine the basic structure of the model.

The $N_{c}=(2,2)$ Multiplets

- Chiral multiplets

${ }^{17}$ In our approach a balanced cohomological field theory [40], [7], [23] is a $N_{c}=$ $(1,1)$ supersymmetric sigma-model in $(0+0)$ dimensions, whose target space can be a general Riemannian space. 
We have the same chiral multiplets introduced in the non-equivariant $N_{c}=(2,2)$ model,

$$
\bar{s}_{ \pm} X^{i}=0 .
$$

We have

$$
\psi_{-}^{i} \underset{s_{+} \searrow}{\stackrel{s_{-}}{\longleftarrow}} X^{i} \stackrel{s_{+}}{\longrightarrow} H^{i} \psi_{+}^{i}
$$

We denote their anti-chiral partners $\left(X^{\bar{i}}, \psi_{ \pm}^{\bar{i}}, H^{\bar{i}}\right)$, which are their Hermitian conjugates.

- Gauge multiplet

The internal consistency of the anti-commutation relations (3.46) determines uniquely the following multiplet

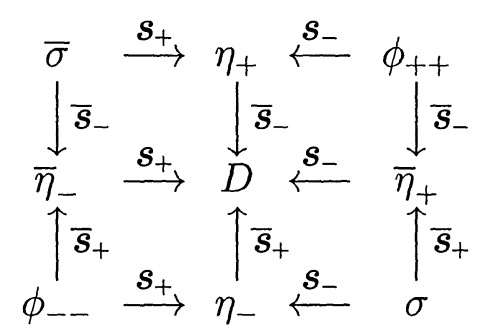

where $D$ is real auxiliary field. All the fields above take values in Lie $(\mathcal{G})$. We call the above multiplet a $N_{c}=(2,2)$ gauge multiplet since it originated from the $\mathcal{G}$ action on $X$. Remark that $\sigma$ is twisted-chiral; i.e.,

$$
s_{+} \sigma=\bar{s}_{-} \sigma=0
$$

- The ghost numbers

The ghost numbers $(p, q)$ of the fields in the gauge multiplet are determined from the assignments (3.45) and the commutation relations (3.46). We set the ghost number of $X^{i}$ to $(0,0)$. For the bosonic fields we have

$$
\begin{array}{cccccccc} 
& \phi & \bar{\phi} & \sigma & \bar{\sigma} & D & X^{i} & H^{i} \\
p & +1 & -1 & +1 & -1 & 0 & 0 & 0 \\
q & +1 & -1 & -1 & +1 & 0 & 0 & 0
\end{array}
$$

- The $\mathcal{R}$-charges 
The $\mathcal{R}$-charges $\left(J_{L}, J_{R}\right)$ of the fields in the gauge multiplet are also determined from the assignments (3.48) and the commutation relations (3.46). We set the $\mathcal{R}$-charges of $X^{i}$ to $(0,0)$. For the bosonic fields we have

$$
\begin{array}{cccccccc} 
& \phi_{++} & \phi_{--} & \sigma & \bar{\sigma} & D & X^{i} & H^{i} \\
J_{L} & 0 & 0 & +1 & -1 & 0 & 0 & 1 \\
J_{R} & 0 & 0 & -1 & +1 & 0 & 0 & 1
\end{array}
$$

The Supersymmetry Transformation Laws

The explicit transformation laws for the fields in the $N_{c}=(2,2)$ gauge multiplet are uniquely determined by the internal consistency

$$
\begin{aligned}
\delta \phi & =i \bar{\epsilon}_{+} \eta_{+}+i \epsilon_{+} \bar{\eta}_{+}, \\
\delta \bar{\phi} & =i \bar{\epsilon}_{-} \eta_{-}+i \epsilon_{-} \bar{\eta}_{-}, \\
\delta \sigma= & -i \bar{\epsilon}_{+} \eta_{-}-i \epsilon_{-} \bar{\eta}_{+}, \\
\delta \bar{\sigma}= & -i \bar{\epsilon}_{-} \eta_{+}-i \epsilon_{+} \bar{\eta}_{-}, \\
\delta \eta_{+}= & +i \epsilon_{+} D-\frac{1}{2} \epsilon_{+}[\sigma, \bar{\sigma}]-\frac{1}{2} \epsilon_{+}\left[\phi_{++}, \phi_{--}\right]-\epsilon_{-}\left[\phi_{++}, \bar{\sigma}\right] \\
\delta \bar{\eta}_{+}= & -i \bar{\epsilon}_{+} D+\frac{1}{2} \bar{\epsilon}_{+}[\sigma, \bar{\sigma}]-\frac{1}{2} \bar{\epsilon}_{+}\left[\phi_{++}, \phi_{--}\right]-\bar{\epsilon}_{-}\left[\phi_{++}, \sigma\right], \\
\delta \eta_{-}= & +i \epsilon_{-} D+\frac{1}{2} \epsilon_{-}[\sigma, \bar{\sigma}]+\frac{1}{2} \epsilon_{-}\left[\phi_{++}, \phi_{--}\right]-\epsilon_{+}\left[\phi_{--}, \sigma\right] \\
\delta \bar{\eta}_{-}= & -i \bar{\epsilon}_{-} D-\frac{1}{2} \bar{\epsilon}_{-}[\sigma, \bar{\sigma}]+\frac{1}{2} \bar{\epsilon}_{-}\left[\phi_{++}, \phi_{--}\right]-\bar{\epsilon}_{+}\left[\phi_{--}, \bar{\sigma}\right], \\
\delta D= & +\frac{1}{2} \bar{\epsilon}_{-}\left[\phi_{++}, \eta_{-}\right]+\frac{1}{2} \bar{\epsilon}_{-}\left[\sigma, \eta_{+}\right]+\frac{1}{2} \bar{\epsilon}_{+}\left[\phi_{--}, \eta_{+}\right]+\frac{1}{2} \bar{\epsilon}_{+}\left[\bar{\sigma}, \eta_{-}\right] \\
& -\frac{1}{2} \epsilon_{-}\left[\phi_{++}, \bar{\eta}_{-}\right]-\frac{1}{2} \epsilon_{-}\left[\bar{\sigma}, \bar{\eta}_{+}\right]-\frac{1}{2} \epsilon_{+}\left[\phi_{--}, \bar{\eta}_{+}\right]-\frac{1}{2} \epsilon_{+}\left[\sigma, \bar{\eta}_{-}\right]
\end{aligned}
$$

where $D$ is an auxiliary field and the commutators are for $\operatorname{Lie}(\mathcal{G})$.

The transformation laws for chiral multiplets are also uniquely determined from the conditions $\bar{s}_{ \pm} X^{i}=0$.

$$
\begin{aligned}
\delta X^{i}= & i \bar{\epsilon}_{+} \psi_{-}^{i}+i \bar{\epsilon}_{-} \psi_{+}^{i} \\
\delta \psi_{+}^{i}= & +\bar{\epsilon}_{+} H^{i}-\epsilon_{-} \phi_{++}^{a} \mathcal{L}_{a}\left(X^{i}\right)-\epsilon_{+} \sigma^{a} \mathcal{L}_{a}\left(X^{i}\right) \\
\delta \psi_{-}^{i}= & -\bar{\epsilon}_{-} H^{i}-\epsilon_{+} \phi_{--}^{a} \mathcal{L}_{a}\left(X^{i}\right)-\epsilon_{-} \bar{\sigma}^{a} \mathcal{L}_{a}\left(X^{i}\right) \\
\delta H^{i}= & +i \epsilon_{-} \phi_{++}^{a} \mathcal{L}_{a}\left(\psi_{-}^{i}\right)+i \epsilon_{-} \eta_{+}^{a} \mathcal{L}_{a}\left(X^{i}\right)-i \epsilon_{-} \bar{\sigma}^{a} \mathcal{L}_{a}\left(\psi_{+}^{i}\right) \\
& -i \epsilon_{+} \phi_{--}^{a} \mathcal{L}_{a}\left(\psi_{+}^{i}\right)-i \epsilon_{+} \eta_{-}^{a} \mathcal{L}_{a}\left(X^{i}\right)+i \epsilon_{+} \sigma^{a} \mathcal{L}_{a}\left(\psi_{-}^{i}\right)
\end{aligned}
$$


where $H^{i}$ are auxiliary fields as in the non-equivariant $N_{c}=(2,2)$ model. The details of the transformation laws above depend on the ways the group $\mathcal{G}$ acts on $X^{i}$. One may have several different chiral multiplets. Their transformation laws are also determined as above once the complex structure and the group action are given for the bosonic fields.

\section{The Fixed Point Equations}

One can never over emphasize the importance of the fixed point theorem of Witten. We have seen many times that the existence of global supersymmetry determine the theories almost uniquely. Such uniqueness becomes stronger as many global supercharges we have.

From the above supersymmetry transformation laws we see that the simultaneous fixed point equations for all the $N_{c}=(2,2)$ are given by

$$
\begin{aligned}
H^{i} & =0 \\
D & =0 \\
\varphi_{m}^{a} \mathcal{L}_{a}\left(X^{i}\right) & =0 \\
{\left[\varphi_{m}, \varphi_{n}\right] } & =0
\end{aligned}
$$

where $\varphi_{m}, m=1, \ldots, 4$ denote the four independent real $\operatorname{Lie}(\mathcal{G})$-valued scalar components of $\phi_{ \pm \pm}, \sigma$ and its Hermitian conjugate $\bar{\sigma}$. The action functional, in many respects, just gives the detailed form of the values of the auxiliary fields $D$ and $H^{i}$. The path integral is localized to the solution space of the above set of equations modulo the $\mathcal{G}$-action. The third equation implies that $\varphi_{m}$ are identically zero if $\mathcal{G}$ act freely on the subset $H^{-1}(0) \cap D^{-1}(0) \subset X$. In such a case the path integral reduces to an integral over the quotient space

$$
\left(H^{-1}(0) \cap D^{-1}(0)\right) / \mathcal{G} .
$$

We call this the effective target space. The $N_{c}=(2,2)$ supersymmetry further implies, as we shall see shortly, that the above space is a Kähler manifold.

If one is interested in evaluating correlation functions of observables invariant only under the supersymmetry generated by $s_{+}$and $\bar{s}_{+}$, the 
path integral is localized to the locus of the following equations

$$
\begin{aligned}
H^{i} & =0, \\
D-\frac{i}{2}[\sigma, \bar{\sigma}] & =0, \\
\bar{\sigma}^{a} \mathcal{L}_{a}\left(X^{i}\right) & =0,
\end{aligned}
$$

and

$$
\begin{aligned}
\phi_{++}^{a} \mathcal{L}_{a}\left(X^{i}\right) & =0, \\
{\left[\phi_{++}, \phi_{--}\right] } & =0, \\
{\left[\phi_{++}, \bar{\sigma}\right] } & =0 .
\end{aligned}
$$

\subsection{Action Functional and Partition Function}

We define the general action functional $S$ by demanding $N_{c}=(2,2)$ supersymmetry, the $\mathcal{G}$-symmetry and the ghost number symmetry. We may, however, not require the $U(1)_{\mathcal{R}}$ symmetry in general. Then $S$ should have the following form ${ }^{18}$

$$
\begin{aligned}
S= & s_{+} s_{-} \bar{s}_{+} \bar{s}_{-} \mathcal{K}\left(X^{i}, X^{\bar{i}}\right)+s_{+} s_{-} \mathcal{W}\left(X^{i}\right)+\bar{s}_{+} \bar{s}_{-} \overline{\mathcal{W}}\left(X^{\bar{i}}\right) \\
& -s_{+} s_{-} \bar{s}_{+} \bar{s}_{-}\langle\sigma, \bar{\sigma}\rangle+\bar{s}_{+} s_{-}\langle t, \sigma\rangle+s_{+} \bar{s}_{-} \operatorname{Tr}\langle\bar{\sigma}, \bar{t}\rangle
\end{aligned}
$$

where all potentials $\mathcal{K}\left(X^{i}, X^{\bar{i}}\right), \mathcal{W}\left(X^{i}\right)$ and its Hermitian conjugate $\overline{\mathcal{W}}\left(X^{\bar{i}}\right)$ are $\mathcal{G}$-invariant and ${ }^{19}$

$$
t=\frac{\theta}{2 \pi}-i \zeta
$$

belongs to the center of $\operatorname{Lie}(\mathcal{G})$. The first line of the action functional (3.61) has the same form as the non-equivariant $N_{c}=(2,2)$ action functional. We remark that the above action functional can be a quite strange object if $\mathcal{K}\left(X^{i}, X^{\bar{i}}\right)$ is non-linear as well as if $X^{i}$ are certain matrices.

\footnotetext{
${ }^{18}$ The total "Kähler" potential $K\left(X^{i}, X^{\bar{i}}\right)-\langle\sigma, \bar{\sigma}\rangle$ can be generalized to an arbitrary $\mathcal{G}$-invariant real functional $\widetilde{\mathcal{K}}\left(X^{i}, X^{\bar{i}} ; \sigma, \bar{\sigma}\right)$. Then we may obtain a model whose effective target space is non-Kähler but has torsion and generally a dilaton. ${ }^{19}$ The theta term plays no roles in the $(0+0)$-dimension we are considering here.
} 
Expanding the action functional above we have the following terms depending on the auxiliary fields

$$
\begin{aligned}
S=\langle D, D\rangle-i\langle D, \mu-\zeta\rangle & +\left\langle g_{i \bar{j}} H^{i}, H^{\bar{j}}\right\rangle \\
& -i\left\langle H^{i}, \partial_{i} \mathcal{W}\right\rangle-i\left\langle H^{\bar{i}}, \partial_{\bar{i}} \overline{\mathcal{W}}\right\rangle+\ldots,
\end{aligned}
$$

where $\mu$ is the $\mathcal{G}$-momentum map on the target space ${ }^{20} X$ as defined earlier in (3.11), $g_{i \bar{j}}:=\partial_{i} \bar{\partial}_{j} \mathcal{K}$ and $\partial_{i} \mathcal{W}=\partial \mathcal{W} / \partial X^{i}$. We integrate out the auxiliary fields $D, H^{i}$ and $H^{\bar{i}}$ by imposing the following algebraic equations of motion

$$
\begin{aligned}
D & =\frac{i}{2}(\mu-\zeta), \\
H^{i} & =i g^{i \bar{j}} \frac{\partial \overline{\mathcal{W}}}{\partial X^{\bar{j}}} .
\end{aligned}
$$

From our general discussion earlier, we see that the path integral is localized to the space of solutions of the following equations

$$
\begin{gathered}
\mu-\zeta=0 \\
\frac{\partial \mathcal{W}}{\partial X^{i}}=0
\end{gathered}
$$

modulo the $\mathcal{G}$-symmetry. In other words the effective target space (3.58) is the symplectic quotient at level $\zeta$ of the critical set $H_{i}^{-1}(0) \subset X$ of the holomorphic potential

$$
\mathcal{M}_{\zeta}:=\left(H_{i}^{-1}(0) \cap \mu^{-1}(\zeta)\right) / \mathcal{G} .
$$

Equivalently $\mathcal{M}_{\zeta}$ is the restriction of $\mathcal{N}_{\zeta}$, the symplectic quotient of $X$ by $\mathcal{G}$, to the critical subset. Those are compatible since $H^{i}$ is $\mathcal{G}$ equivariant as $\mathcal{W}$ and $S$ are $\mathcal{G}$-invariant. Thus $\mathcal{M}_{\zeta}$ is a Kähler manifold, provided that $\zeta$ is generic. Note that the space of all bosonic fields is much bigger than $X$ due to the additional affine space of four real scalars $\varphi^{m}, m=1, \ldots, 4$. The path integral is localized, in addition to (3.65), to the space of solutions of

$$
\left[\varphi^{m}, \varphi^{n}\right]=0, \quad \varphi_{m}^{a} \mathcal{L}_{a}\left(X^{i}\right)=0,
$$

modulo the gauge symmetry. As the basic principle of the equivariant cohomology $\varphi_{m}=0$ if $\mathcal{G}$ acts freely while, otherwise, there is something else.

\footnotetext{
${ }^{20} \mathrm{~A}$ better terminology is to regard $X$ as the space of all $X^{i}$ s.
} 
Now we assume that $\mathcal{M}_{\zeta}$ is smooth. Then our model is equivalent to the non-equivariant $N_{c}=(2,2)$ model with target space $\mathcal{M}_{\zeta}$. Thus the partition function is the Euler characteristic of the effective target space;

$$
Z=\chi\left(T \mathcal{M}_{\zeta}\right)=\chi\left(\mathcal{M}_{\zeta}\right)
$$

A beautiful fact is that our initial target space $X$ may be infinite dimensional with an infinite dimensional group $\mathcal{G}$ acting on it, while the final target space $\mathcal{M}_{\zeta}$ can be finite dimensional.

\subsubsection{The Geometry of Effective Target Space}

It is obvious that the group action preserves the condition $H_{i}=0$ and the subvariety $H_{i}^{-1}(0) \subset X$ inherits the complex and Kähler structures by restriction. The quotient space $\mathcal{M}_{\zeta}$ inherits the Kähler structure from $H_{i}^{-1}(0)$ by the restrictions and the reduction.

If $\zeta$ takes on a generic value, the group $\mathcal{G}$ acts freely and $\mathcal{M}_{\zeta}$ is a smooth Kähler manifold. For such a case the model can be identified with the non-linear non-equivariant $N_{c}=(2,2)$ model in Sect. 2.2.2 with target space $\mathcal{M}_{\zeta}$. This property is equivalent to the property of equivariant cohomology that the equivariant cohomology is the ordinary cohomology of the quotient space if it is smooth.

For non-generic $\zeta$ the quotient space develops singularities or even may not exist at all. For such cases however one always has some extra degrees of freedom not described by the moduli space, due to the extension of $X / \mathcal{G}$ to $X_{\mathcal{G}}$. Those extra degrees of freedom are represented by the solutions of (3.67) modulo gauge symmetry. The first equation in (3.67) show that no such a solution exists if the $\mathcal{G}$ action act freely, without fixed points, on $X$. If there are solutions they span an affine space ${ }^{21}$ which looks like a symmetric products of $\mathbb{R}^{4}$.

The beautiful relation between the symplectic and geometrical invariant theory (GIT) quotients also is an important part of the story [41], [42], [38]. The essential point is that the condition $H_{i}=0$ is preserved by the complexified group action $\mathcal{G}^{\mathbb{C}}$, while the condition $D=0$ is only preserved by the real group action. Thus we may consider

\footnotetext{
${ }^{21}$ We will relate those degrees of freedom, in certain cases, with the degrees transverse to the D-brane world volume in the bulk.
} 
a complex quotient $H_{i}^{-1}(0) / \mathcal{G}^{\mathbb{C}}$ and try to compare with the real quotient $\left(H_{i}^{-1}(0) \cap D^{-1}(0)\right) / \mathcal{G}$. In general there can be $\mathcal{G}^{\mathbb{C}}$-orbits in $H_{i}^{-1}(0)$ which contain several $\mathcal{G}$ orbits in $H_{i}^{-1}(0) \cap D^{-1}(0)$. Thus we need to consider a suitable subset in $H_{i}^{-1}(0)$ for which a $\mathcal{G}^{\mathbb{C}}$-orbit contains exactly one solution of the equation $D=0$. Then the real equation $D=0$ can be identified with the gauge fixing condition of the complex gauge symmetry of the complex equations $H_{i}=0$.

The complex gauge group in general does not act freely on the submanifold $H_{i}^{-1}(0)$, so that taking the quotient directly would lead to unwanted singularities. One first removes such obvious bad points $B$. However there are subsets in $\left(H_{i}^{-1}(0)-B\right)$ which can be arbitrarly close to $B$ by $\mathcal{G}^{\mathbb{C}}$ action. One call a point in $H_{i}^{-1}(0)$ semi-stable if the closure of its $\mathcal{G}^{\mathbb{C}}$ orbit does not contain $B$. Let $H_{i}^{-1}(0)_{s s}$ be the semi-stable subset of $H_{i}^{-1}(0)$. Now the beautiful fact is that the complex quotient $H_{i}^{-1}(0)_{s s} / \mathcal{G}^{\mathbb{C}}$ contains the symplectic quotient $\mathcal{M}_{0}$ as open subset. A stable orbit is a semi-stable orbit if the points of the orbit have at most finite stabilizers under the real $\mathcal{G}$ action. Then the various symplectic quotients $\mathcal{M}_{\zeta}$ can be identified with the quotient space $H^{-1}(0)_{s} / \mathcal{G}^{\mathbb{C}}$ in dense open subset. Thus we have

$$
H_{i}^{-1}(0)_{s s} / \mathcal{G}^{\mathbb{C}} \supset \mathcal{M}_{\zeta} \supset H^{-1}(0)_{s} / \mathcal{G}^{\mathbb{C}}
$$

The first relation implies that we have a natural compactification of $\mathcal{M}_{\zeta}$ by taking the closure in $H_{i}^{-1}(0)_{s s} / \mathcal{G}^{\mathbb{C}}$. The second relation implies that the various symplectic quotients $\mathcal{M}_{\zeta}$ are birational with each others.

\subsection{Generalization to Equivariant $N_{c}=(2,0)$ Model}

Now we consider the equivariant extension of the $N_{c}=(2,0)$ model introduced in Sect. 2.3 or, equivalently, the generalization of the equivariant $N_{w s}=(2,2)$ model in the previous section. We consider the same group $\mathcal{G}$ acting on $X$ as before but now we allow the $\mathcal{G}$ action to extend to a Hermitian holomorphic vector bundle $\mathbb{E} \rightarrow X$ preserving the Hermitian structure. We have two supercharges $\boldsymbol{s}_{+}$and $\overline{\boldsymbol{s}}_{+}$, isomorphic to the differentials of $\mathcal{G}$-equivariant Dolbeault cohomology as in the equivariant toy model in Sect. 3.1;

$$
s_{+}^{2}=0, \quad\left\{s_{+}, \bar{s}_{+}\right\}=-i \phi_{++}^{a} \mathcal{L}_{a}, \quad \bar{s}_{+}^{2}=0 .
$$


Comparing with the non-equivariant counterpart, the equivariant $N_{c}=(2,0)$ model has essentially one addition structure that the path integral is further localized to the vanishing locus $\mu^{-1}(\zeta)$ of $\mathcal{G}$-moment map. If $\mathcal{G}$ acts freely on $\mu^{-1}(\zeta)$ the model reduce to a standard $N_{c}=$ $(2,0)$ model associated with the symplectic quotients. The observables of the model are given by $\mathcal{G}$-equivariant closed differential forms, after the parity changes, as our equivariant toy model. If $\mathcal{G}$ acts freely on $\mu^{-1}(\zeta)$ those observables become ordinary closed differential form on the symplectic quotient. Comparing with our equivariant toy model the additional structure is that the path integral is further localized to the locus of vanishing holomorphic sections on $\mathbb{E}$. We will use such property to define a more general hybrid $N_{c}=(2,0)$ model. Following the discussion in Sect. 3.2 the model is related with $N_{w s}=(2,0)$ world-sheet gauged sigma-model in $(1+1)$ dimensions by dimensional reduction [18].

\subsubsection{Basic Structures}

We may follow exactly the same route as we followed to arrive at the non-equivariant $N_{c}=(2,0)$ model from the non-equivariant $N_{c}=(2,2)$ models.

First we write the $N_{c}=(2,2)$ action functional $S$ (3.61) in a form such that only the $s_{+}$and $\bar{s}_{+}$are manifest - compare with (2.69) -

$$
\begin{aligned}
S(\zeta)= & -s_{+} \bar{s}_{+}\left(\left\langle\phi_{--}, \mu\left(X^{i}, X^{\bar{i}}\right)-\zeta\right\rangle-\left\langle\eta_{-}, \bar{\eta}_{-}\right\rangle\right. \\
& \left.+\left\langle g_{i \bar{j}}\left(X^{k}, X^{\bar{k}}\right) \psi_{-}^{i}, \psi_{-}^{\bar{j}}\right\rangle\right) \\
& +i s_{+}\left\langle\psi_{-}^{i}, V_{i}\left(X^{j}\right)\right\rangle+i \bar{s}_{+}\left\langle\psi_{-}^{\bar{i}}, V_{\bar{i}}\left(X^{\bar{j}}\right)\right\rangle
\end{aligned}
$$

where $V_{i}=\partial \mathcal{W} / \partial X^{i}$. Similarly we disconnect the diagram (3.50) by removing the link $s_{-}$,

$$
\begin{array}{lll}
\psi_{-}^{i} \underset{s_{+} \searrow}{ }{ }^{X^{i}} \stackrel{s_{+}}{\longrightarrow} \psi_{+}^{i} \\
& &
\end{array}
$$

Now we regard the above as two independent sets of multiplets. Then 
we rename various fields as follows, exactly the same as earlier (2.71)

$$
\begin{array}{lll}
\psi_{-}^{i} \rightarrow \chi_{-}^{\alpha}, & H^{i} \rightarrow H^{\alpha}, & V_{i} \rightarrow \mathfrak{S}_{\alpha}\left(X^{j}\right), \\
\psi_{-}^{\bar{i}} \rightarrow \chi_{-}^{\bar{\alpha}}, & H^{\bar{i}} \rightarrow H^{\bar{\alpha}}, & V_{\bar{i}} \rightarrow \mathfrak{S}_{\bar{\alpha}}\left(X^{\bar{j}}\right), \\
& g_{i \bar{j}} \rightarrow h_{\alpha \bar{\beta}}\left(X^{i}, X^{\bar{i}}\right), &
\end{array}
$$

where the new indices run as $\alpha, \beta=1, \ldots, r$ and we maintain the Hermiticity of $h_{\alpha \bar{\beta}}$. The $N_{c}=(2,0)$ multiplets $\left(X^{i}, \psi_{+}^{i}\right)$ are holomorphic, i.e., $\overline{\boldsymbol{s}}_{+} X^{i}=0$. We call the multiplets $\left(\chi_{-}^{\alpha}, H^{\alpha}\right)$ Fermi multiplets. We also disconnect the diagram (3.51) for the $N_{c}=(2,2)$ gauge multiplet by removing the links $\boldsymbol{s}_{-}$and $\overline{\boldsymbol{s}}_{-}$,

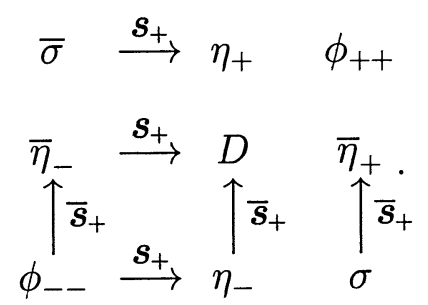

Note that $\bar{\sigma}$ is holomorphic, i.e., $\overline{\boldsymbol{s}}_{+} \bar{\sigma}=0$. Thus the $N_{c}=(2,0)$ multiplet $\left(\bar{\sigma}, \eta_{+}\right)$is another holomorphic multiplet, while their Hermitian conjugates $\left(\sigma, \bar{\eta}_{+}\right)$form an anti-holomorphic multiplet. We may simply remove them, or keep them as they are still valued in $\operatorname{Lie}(\mathcal{G})$, or just regard them as another holomorphic multiplet supplementing the multiplets $\left(X^{i}, \psi_{+}^{i}\right){ }^{22}$ We call the multiplet $\left(\phi_{--}, \eta_{-}, \bar{\eta}_{-}, D\right) N_{c}=(2,0)$ gauge multiplet taking values in $\operatorname{Lie}(\mathcal{G})$.

Now we consider the transformation laws for the $\boldsymbol{s}_{+}$and $\overline{\boldsymbol{s}}_{+}$supersymmetry. For the holomorphic multiplets $\left(X^{i}, \psi_{+}^{i}\right)$, i.e., $\overline{\boldsymbol{s}}_{+} X^{i}=0$, and their conjugates we have

$$
\begin{aligned}
& \boldsymbol{s}_{+} X^{i}=i \psi_{+}^{i}, \quad \boldsymbol{s}_{+} \psi_{+}^{i}=0, \\
& \overline{\boldsymbol{s}}_{+} X^{i}=0, \quad \overline{\boldsymbol{s}}_{+} \psi_{+}^{i}=\phi_{++}^{a} \mathcal{L}_{a} X^{i}, \quad \boldsymbol{s}_{+} \phi_{++}=0, \\
& s_{+} X^{\bar{i}}=0, \quad s_{+} \psi_{+}^{\bar{i}}=\phi_{++}^{a} \mathcal{L}_{a} X^{\bar{i}}, \quad \bar{s}_{+} \phi_{++}=0 \\
& \overline{\boldsymbol{s}}_{+} X^{\bar{i}}=i \psi_{+}^{\bar{i}}, \quad \overline{\boldsymbol{s}}_{+} \psi_{+}^{\bar{i}}=0,
\end{aligned}
$$

\footnotetext{
${ }^{22}$ It is our convention that all the holomorphic multiplets are collectively denoted as $\left(X^{i}, \psi_{+}^{i}\right)$ where each multiplet may transform differently under $\mathcal{G}$ and other global symmetries. We also denote $X$ as the space of all $X^{i}$ 's.
} 
which are, of course, the same as (3.7). The transformation laws for Fermi multiplets $\left(\chi_{-}^{\alpha}, H^{\alpha}\right)$ and their conjugates are given by

$$
\begin{array}{ll}
\boldsymbol{s}_{+} \chi_{-}^{\alpha}=-H^{\alpha}, & \boldsymbol{s}_{+} H^{\alpha}=0, \\
\bar{s}_{+} \chi_{-}^{\alpha}=\mathfrak{J}^{\alpha}\left(X^{i}\right), & \bar{s}_{+} H^{\alpha}=-i \phi_{++}^{a} \mathcal{L}_{a} \chi_{-}^{\alpha}+i \psi_{+}^{i} \partial_{i} \mathfrak{J}^{\alpha}\left(X^{j}\right), \\
\boldsymbol{s}_{+} \chi_{-}^{\bar{\alpha}}=\mathfrak{J}^{\bar{\alpha}}\left(X^{\bar{i}}\right), & \boldsymbol{s}_{+} H^{\bar{\alpha}}=-i \phi_{++}^{a} \mathcal{L}_{a} \chi_{-}^{\bar{\alpha}}+i \psi_{+}^{\bar{i}} \partial_{\bar{i}} \mathfrak{J}^{\bar{\alpha}}\left(X^{\bar{j}}\right), \\
\bar{s}_{+} \chi_{-}^{\bar{\alpha}}=-H^{\bar{a}}, & \bar{s}_{+} H^{\bar{\alpha}}=0,
\end{array}
$$

where $\partial_{\bar{i}} \mathfrak{J}^{\alpha}\left(X^{j}\right)=0$. Note that $\bar{s}_{+} \chi_{-}^{\alpha} \neq 0$ but rather equals $\mathfrak{J}^{\alpha}\left(X^{i}\right)$, while the above transformation laws are consistent, since $\bar{s}_{+}^{2} \chi_{-}^{\alpha}=$ $\overline{\boldsymbol{s}}_{+} \mathfrak{J}^{\alpha}\left(X^{i}\right)=0$, with the commutation relations (3.70). Finally the transformation laws for the $N_{c}=(2,0)$ gauge multiplet $\left(\phi_{--}, \eta_{-}, \bar{\eta}_{-}\right)$ are given by

$$
\begin{aligned}
s_{+} \eta_{-} & =0 \\
s_{+} \phi_{--}=i \eta_{-}, & \bar{s}_{+} \eta_{-}=+i D+\frac{1}{2}\left[\phi_{++}, \phi_{--}\right] \\
\bar{s}_{+} \phi_{--}=i \bar{\eta}_{-}, & s_{+} \bar{\eta}_{-}=-i D+\frac{1}{2}\left[\phi_{++}, \phi_{--}\right] \\
& \bar{s}_{+} \bar{\eta}_{-}=0 .
\end{aligned}
$$

The general $N_{c}=(2,0)$ action functional, with the vanishing ghost number, is given by the following form ${ }^{23}$

$$
\begin{aligned}
S(\zeta)= & -s_{+} \bar{s}_{+}\left(\left\langle\phi_{--}, \mu-\zeta\right\rangle-\left\langle\eta_{-}, \bar{\eta}_{-}\right\rangle+\left\langle h_{\alpha \bar{\beta}} \chi_{-}^{\alpha}, \chi_{-}^{\bar{\alpha}}\right\rangle\right) \\
& +i s_{+}\left\langle\chi_{-}^{\alpha}, \mathfrak{S}^{\alpha}\left(X^{i}\right)\right\rangle+i \bar{s}_{+}\left\langle\chi_{-}^{\bar{\alpha}}, \mathfrak{S}^{\bar{\alpha}}\left(X^{\bar{i}}\right)\right\rangle .
\end{aligned}
$$

Here $h_{\alpha \bar{\beta}}\left(X^{i}, X^{\bar{i}}\right)$ is a Hermitian structure on a Hermitian vector bundle $\mathbb{E}$ over $X, \mathfrak{S}^{\alpha}\left(X^{i}\right)$ a holomorphic section and $\mu\left(X^{i}, X^{\bar{i}}\right)$ is the $\mathcal{G}$ momentum map on $X$. Note that $N_{c}=(2,0)$ symmetry of the above action functional is not obvious due to the second line in (3.78). For example the $\overline{\boldsymbol{s}}_{+}$supersymmetry of the term $\boldsymbol{s}_{+}\left\langle\chi_{-}^{\alpha}, \mathfrak{S}_{\alpha}\right\rangle$ is not obvious if $\mathfrak{J}\left(X^{i}\right) \neq 0$ due to the transformation law $\bar{s}_{+} \chi_{-}^{\alpha}=\mathfrak{J}\left(X^{i}\right)$. The condition that the action functional $S(\zeta)$ has $N_{c}=(2,0)$ is

$$
\overline{\boldsymbol{s}}_{+}\left\langle\chi_{-}^{\alpha}, \mathfrak{S}^{\alpha}\left(X^{i}\right)\right\rangle=\left\langle\mathfrak{J}^{\alpha}\left(X^{i}\right), \mathfrak{S}^{\alpha}\left(X^{i}\right)\right\rangle=0 .
$$

\footnotetext{
${ }^{23}$ The repeated indices are summed over unless otherwise stated.
} 
Let us summarize the basic structure of an equivariant $N_{c}=(2,0)$ model.

1. A complex Kähler target space $X$ with a $\mathcal{G}$ symmetry as an isometry. These data determine holomorphic multiplets and gauge multiplets as well as their transformation laws and $\mathcal{G}$-equivariant momentum map $\mu: X \rightarrow \operatorname{Lie}(\mathcal{G})^{*}$.

2. A Hermitian holomorphic vector bundle $\mathbb{E} \rightarrow X$ over the target space $X$ with the $\mathcal{G}$ action preserving the Hermitian structure. We may have up to two $\mathcal{G}$-equivariant holomorphic sections $\mathfrak{S}$ and $\mathfrak{J}$ orthogonal with each others by a natural non-degenerated $\mathcal{G}$ invariant parings. Those sections determine Fermi multiplets and their transformation laws.

Given the data above, we have an unique family of equivariant $N_{c}=$ $(2,0)$ models parameterized by the FI term $\zeta$.

\subsubsection{The Path Integrals}

Expanding the action functional $S$ (3.78) we have the following terms depending on the auxiliary fields $D, H^{\alpha}$ and $H^{\bar{\alpha}}$,

$$
\begin{aligned}
S=\langle D, D\rangle-\langle D, \mu-\zeta\rangle+ & \left\langle h_{\alpha \bar{\beta}} H^{\alpha}, H^{\bar{\beta}}\right\rangle \\
& -i\left\langle H^{\alpha}, \mathfrak{S}^{\alpha}\right\rangle-i\left\langle H^{\bar{\alpha}}, \mathfrak{S}^{\bar{\alpha}}\right\rangle+\ldots
\end{aligned}
$$

We integrate the auxiliary fields out by imposing the following algebraic equations of motion,

$$
\begin{aligned}
D & =\frac{1}{2}(\mu-\zeta), \\
H_{\alpha} & =i h_{\alpha \bar{\beta}} \mathfrak{S}^{\bar{\beta}} .
\end{aligned}
$$

From our general discussion earlier, we see that the bosonic part of the path integral reduces to an integral over the space of solutions of the following equations,

$$
\begin{aligned}
\mathfrak{J}^{\alpha}\left(X^{i}\right) & =0, \\
\mathfrak{S}_{\alpha}\left(X^{i}\right) & =0, \\
\mu-\zeta & =0,
\end{aligned}
$$


and

$$
\begin{aligned}
\phi_{++}^{a} \mathcal{L}_{a} X^{i} & =0 \\
{\left[\phi_{++}, \phi_{--}\right] } & =0
\end{aligned}
$$

modulo $\mathcal{G}$-symmetry.

Now we examine the properties of the path integral in some detail by applying the fixed point theorem of Witten. For simplicity assume that the space $X$ and the Hermitian holomorphic bundle $E$ are flat. We also turn off the section $\mathfrak{J}^{\alpha}$, keeping $\mathfrak{S}$ only. Then the fixed point locus of the $\boldsymbol{s}_{+}$and $\overline{\boldsymbol{s}}_{+}$supersymmetry is the symplectic quotient $\mathcal{M}_{\zeta}$ of $\mathfrak{S}_{\alpha}^{-1}(0) \subset X$ by $\mathcal{G}$;

$$
\mathcal{M}_{\zeta}=\left(\mu^{-1}(\zeta) \cap \mathfrak{S}_{\alpha}^{-1}(0)\right) / \mathcal{G}
$$

We have the same set of observables as in the equivariant toy model, given by $\boldsymbol{s}_{+}$and $\overline{\boldsymbol{s}}_{+}$closed $\mathcal{G}$-equivariant differential forms $\widehat{\mathcal{O}}^{r, s}$ wite ghost numbers $(r, s)$.

The explicit expression of the action functional is

$$
\begin{aligned}
S^{\prime}= & D^{2}+\sum\left|H_{\alpha}\right|^{2}-\frac{1}{4}\left[\phi_{++}, \phi_{--}\right]^{2}-i\left[\phi_{++}, \eta_{-}\right]_{a} \bar{\eta}_{-}^{a} \\
& -\bar{\eta}_{-}^{a} \partial_{i} \mu_{a} \psi_{+}^{i}-\eta_{-}^{a} \partial_{\bar{i}} \mu_{a} \psi_{+}^{\bar{i}}+\chi_{-}^{\alpha} \partial_{i} \mathfrak{S}_{\alpha} \psi_{+}^{i}+\chi_{-}^{\bar{\alpha}} \partial_{i} \mathfrak{S}_{\bar{\alpha}} \psi_{+}^{\bar{i}} \\
& -i h_{\alpha \bar{\beta}} \phi_{++}^{a} \mathcal{L}_{a} \chi_{-}^{\alpha} \chi_{-}^{\bar{\beta}}+i \phi_{--}^{a}\left(\phi_{++}^{b} \partial_{\bar{i}} \mu_{a} V_{b}^{\bar{i}}+\partial_{i} \partial_{\bar{j}} \mu_{a} \psi_{+}^{i} \psi_{+}^{\bar{j}}\right),
\end{aligned}
$$

where $V_{b}^{\bar{i}}=\mathcal{L}_{a} X^{\bar{i}}$. In doing the path integral one replaces all fields yb their zero-modes. The zero-modes of the fermions are solutions of the following equations

$$
\begin{array}{rlrl}
\partial_{\bar{i}} \mu_{a} \psi_{+}^{\bar{i}} & =0, & & \eta_{-}^{a} \partial_{\bar{i}} \mu_{a}=0, \\
\partial_{\bar{i}} \mathfrak{S}_{\bar{\alpha}} \psi_{+}^{\bar{i}}=0, & & \chi^{\bar{\alpha}} \partial_{\bar{i}} \mathfrak{S}_{\bar{\alpha}}=0 .
\end{array}
$$

The above equations implies that the net ghost number violation $\triangle$ in the path integral measure due to fermionic zero-modes of $\left(\psi_{+}^{\bar{i}}, \chi_{-}^{\bar{\alpha}}, \eta_{-}\right)$ always equals

$$
\triangle=n-r-\operatorname{dim} \mathcal{G} .
$$

We call $\triangle$ the virtual complex dimension of $\mathcal{M}_{\zeta}$. From the equations $\partial_{\bar{i}} \mu_{a} \psi_{+}^{\bar{i}}=0$ we have the following integrability condition

$$
\phi_{++}^{b} \partial_{\bar{i}} \mu_{a} V_{b}^{\bar{i}}+\partial_{i} \partial_{\bar{j}} \mu_{a} \psi_{+}^{i} \psi_{+}^{\bar{j}}=0
$$


which is also the $\phi_{--}^{a}$ equation of motion. This implies that one can simply replace $\phi_{++}^{a}$ with the solutions of the above. Such an argument can not be justified if there are zero-modes of $\phi_{++}^{a}$, which are given by the non-trivial solutions of (3.83), for instance $\phi_{++}^{b} V_{b}^{\bar{i}}=0$.

Here we specialize to the case that $\mathcal{G}$ acts freely, thus there are no zero-modes of $\eta_{-}^{a}$ and $\phi_{ \pm \pm}^{a}$. Then the only non-trivial term in teh action functional $S^{\prime}$ in the $s_{+}$and $\bar{s}_{+}$invariant neighborhood $\mathcal{C}$ of the fixed point locus is

$$
\left.S^{\prime}\right|_{\mathcal{C}}=-\left.i h_{\alpha \bar{\beta}} \phi_{++}^{a} \mathcal{L}_{a} \chi_{-}^{\alpha} \chi_{-}^{\bar{\beta}}\right|_{\mathcal{C}}
$$

Using (3.88) we can solve $\phi_{++}^{a}$ in terms of the zero-modes $\left(u^{\bar{i}^{\prime}}, \widetilde{\psi}_{+}^{i^{\prime}}, \widetilde{\chi}_{-}^{\bar{\alpha}^{\prime}}\right)$ of $\left(X^{\bar{i}}, \psi_{+}^{\bar{i}}, \chi_{-}^{\bar{\alpha}}\right)$

$$
\left\langle\phi_{++}^{a}\left(u^{i^{\prime}}, u^{\bar{i}^{\prime}}\right)\right\rangle=-\left(\partial_{\bar{\ell}^{\prime}} \mu_{b} V_{a}^{\bar{\ell}^{\prime}}\right)^{-1} \partial_{i^{\prime}} \partial_{\bar{j}^{\prime}} \mu_{b} \widetilde{\psi}_{+}^{i^{\prime}} \widetilde{\psi_{+}^{j^{\prime}}}
$$

where the primed indices above are understood to label independent zero-modes $-\bar{i}^{\prime}=1, \ldots, n^{\prime}, \bar{\alpha}^{\prime}=1, \ldots, r^{\prime}$, with the condition

$$
\triangle=n^{\prime}-r^{\prime}=n-r-\operatorname{dim} \mathcal{G} .
$$

Then we may write

$$
\left.S^{\prime}\right|_{\mathcal{C}}=-\mathcal{F}\left(u^{\ell^{\prime}}, u^{\bar{\ell}^{\prime}}\right)_{i^{\prime} \bar{j}^{\prime} \alpha^{\prime} \bar{\beta}^{\prime}} \widetilde{\psi}_{+}^{i^{\prime}} \widetilde{\psi}_{+}^{\bar{j}^{\prime}} \widetilde{\chi}_{-}^{\alpha^{\prime}} \widetilde{\chi}_{-}^{\bar{\beta}^{\prime}}
$$

where $\mathcal{F}_{i^{\prime} \bar{j}^{\prime} \alpha^{\prime} \bar{\beta}^{\prime}} \widetilde{\psi}_{+}^{i_{+}^{\prime}} \widetilde{\psi}_{+}^{\widetilde{j}^{\prime}}$ can be interpreted as the curvature two-form of the anti-ghost bundle $\mathbb{V}$ over $\mathcal{M}_{\zeta}$. Consequently the path integral reduces to

$$
\begin{aligned}
\left\langle\prod_{m=1}^{k} \widehat{\mathcal{O}}^{r_{m}, s_{m}}\right\rangle= & \int_{\mathcal{M}_{\zeta}} \prod_{\gamma^{\prime}=1}^{r^{\prime}} d \widetilde{\chi}_{-}^{\gamma^{\prime}} d \widetilde{\chi}_{-}^{\bar{\gamma}^{\prime}} \prod_{\ell^{\prime}=1}^{n^{\prime}} d u^{\ell^{\prime}} d u^{\bar{\ell}^{\prime}} d \widetilde{\psi}_{+}^{\ell^{\prime}} \widetilde{\psi}_{+}^{\ell^{\prime}} \\
& \times \exp \left(\mathcal{F}_{i^{\prime} \bar{j}^{\prime} \alpha^{\prime} \bar{\beta}^{\prime}} \widetilde{\psi}_{+}^{i^{\prime}} \widetilde{\psi}_{+}^{\widetilde{\psi}^{\prime}} \widetilde{\chi}_{-}^{\alpha^{\prime}} \widetilde{\chi}_{-}^{\bar{\beta}^{\prime}}\right) \prod \widetilde{\mathcal{O}}^{r_{m}, s_{m}}
\end{aligned}
$$

where $\widehat{\widetilde{\mathcal{O}}}$ denote the expression of an observable $\widehat{\mathcal{O}}$ in terms of zeromodes and $\left\langle\phi_{++}\right\rangle$. The necessary condition for a non-vanishing correlation function is

$$
\sum_{m=1}^{k}\left(r_{m}, s_{m}\right)=(\triangle, \triangle) .
$$


Let us first assume that the section is generic and $\mathcal{G}$ acts freely on $\mathfrak{S}_{\alpha}^{-1}(0) \subset X$. Then $\mathcal{M}_{\zeta}$ is a smooth non-linear Kähler manifold with complex dimensions

$$
\operatorname{dim}_{\mathbb{C}} \mathcal{M}_{\zeta}=\triangle=n-r-\operatorname{dim} \mathcal{G}
$$

The above counting goes as follows. Since $\mathfrak{S}_{\alpha}, \alpha=1, \ldots, r$, are generic they are all independent and transverse. Thus the condition $\mathfrak{S}_{\alpha}=0$ cuts out a complex $(n-r)$ smooth submanifold inside the complex $n$-dimensional ambient space $X$. On the subspace we further impose $\operatorname{dim} \mathcal{G}$ real equations $\mu_{a}-\zeta=0$ and take the quotient by the free $\mathcal{G}$ action. Now we do not have zero-modes of $\chi_{-}$and the path integral becomes

$$
\begin{aligned}
\left\langle\prod_{m=1}^{k} \widehat{\mathcal{O}}^{r_{m}, s_{m}}\right\rangle & =\int_{\mathcal{M}_{\zeta}} \prod_{\ell^{\prime}=1}^{\Delta} d u^{\ell^{\prime}} d u^{\bar{\ell}^{\prime}} d \widetilde{\psi}_{+}^{\ell^{\prime}} \widetilde{\psi}_{+}^{\bar{\ell}^{\prime}} \prod_{m} \widehat{\widehat{\widetilde{\mathcal{O}}}}^{r_{m}, s_{m}} \\
& =\int_{\mathcal{M}_{\zeta}} \widetilde{\mathcal{O}}^{r_{1}, s_{1}} \wedge \ldots \wedge \widetilde{\mathcal{O}}^{r_{k}, s_{k}}
\end{aligned}
$$

A non-generic situation arises when $\mathfrak{S}_{\alpha^{\prime}}, \alpha^{\prime}=1, \ldots, r^{\prime}$, are linearly dependent to the remaining sections. Then the complex dimension of $\mathcal{M}_{\zeta}$ is given by $n^{\prime}=\Delta+r^{\prime}$. The resulting space is smooth if the linearly independent components of the section are transverse. We have $r^{\prime} \chi_{-}$ zero-modes which span the anti-ghost bundle $\mathbb{V}$ over $\mathcal{M}_{\zeta}$. The path integral becomes

$$
\left\langle\prod_{m=1}^{k} \widehat{\mathcal{O}}^{r_{m}, s_{m}}\right\rangle=\int_{\mathcal{M}_{\zeta}} e(\mathbb{V}) \wedge \widetilde{\mathcal{O}}^{r_{1}, s_{1}} \wedge \ldots \wedge \widetilde{\mathcal{O}}^{r_{k}, s_{k}}
$$

A beautiful fact about this is that $(X, \mathbb{E}, \mathcal{G})$ can be all infinite dimensional while the space $\mathcal{M}_{\zeta}$ can be a finite dimensional space. In particular $X$ can be a certain function space defined by the space of all fields of a certain gauge field theory on a manifold $M$. Then the integral we are dealing with is a genuine path integral of a non-trivial quantum field theory on $M$, while the path integral eventually reduces to an ordinary integral on a smooth finite dimensional space $\mathcal{M}_{\zeta}$. The above is a key principle underlying cohomological field theory [1], [13]. In principle the above path integral formalism is well-defined regardless of the properties the moduli space $\mathcal{M}_{\zeta}$. 
Finally we remark that a proper mathematical interpretation of our formalism may be a certain equivariant version of Fulton and MacPherson's intersection theory [25].

\section{Generalizations}

In this section we consider three geneneralizations of an equivariant $N_{c}=(2,0)$ model.

\subsection{Deformation to Holomorphic $N_{c}=(2,0)$ Model}

In this subsection we introduce hybrid $\mathcal{N}_{c}=(2,0)$ model of the equivariant $N_{c}=(2,0)$ mode and the equivariant toy model in Sect. 2.2. The resulting hybrid model will have much better behavior than the original model when the effective target space $\mathcal{M}_{\zeta}$ has singularities. To motivate such a model we first compare the two models.

First of all both the models have the same supersymmetry generated by $s_{+}$and $\bar{s}_{+}$, which are the differentials of equivariant Dolbeault cohomology after the parity change. Secondly both the models share the same holomorphic multiplets $\left(X^{i}, \psi_{+}^{i}\right)$ and their Hermitian conjugates, which are anti-holomorphic multiplets $\left(X^{\bar{i}}, \psi_{+}^{\bar{i}}\right)$. Thus they share the same observables, given by equivariantly closed differential forms on $X$, the space of all $X^{i}$, after the parity change.

A difference is that the equivariant $N_{c}=(2,0)$ model has the additional Fermi multiplets $\left(\chi_{-}^{\alpha}, H^{\alpha}\right)$ and their Hermitian conjugates $\left(\chi_{-}^{\bar{\alpha}}, H^{\bar{\alpha}}\right)$. The roles of the Fermi multiplets are to restrict the (path) integral over $X$ to the subspace defined by $\mathfrak{J}_{\alpha}^{-1}(0) \cap \mathfrak{S}_{\alpha}^{-1}(0) \subset X$. For convenience we denote this subspace by $X^{1,1} \subset X$. We saw that the path integral of the $N_{c}=(2,0)$ model is localized to the symplectic quotients $\mathcal{M}_{\zeta}=\left(X^{1,1} \cap \mu^{-1}(\zeta)\right.$ of $X^{1,1}$ by $\mathcal{G}$. Now we consider an equivariant toy model whose initial target space is $X^{1,1}$. Then, its path integral is also localized to the same space $\mathcal{M}_{\zeta}$, provided that we set $\varepsilon=0$ in the action functional $S(\zeta, \varepsilon)$ defined by (3.37). We also note that the partition function of the above equivariant toy model is the expectation value of $\exp \left(\widehat{\varpi}_{\mathcal{G}}\right)$ evaluated by the $N_{c}=(2,0)$ model, 
where

$$
\widehat{\varpi}_{\mathcal{G}}:=S(\zeta, 0)=i\left\langle\phi_{++}, \mu-\zeta\right\rangle+i g_{i \bar{j}} \psi_{+}^{i} \psi_{+}^{\bar{i}} .
$$

The first term above is irrelevant as the path integral of the $N_{c}=(2,0)$ is localized the to the locus $\mu-\zeta=0$, while the second term above becomes the Kähler from $\widetilde{\varpi}$ on $\mathcal{M}_{\zeta}$. We note that it is the $N_{c}=$ $(2,0)$ gauge multiplet $\left(\phi_{--}, \eta_{-}, \bar{\eta}_{-}, D\right)$, which is responsible for such a localization. One the other hand, the above is the action functional of the equivariant toy model on $X^{1,1}$ and the integration over $\phi$ localizes the path integral by a delta function supported on $\mathcal{M}_{\zeta}$ in $X^{1,1}$. Note that $\mathcal{M}_{\zeta}=\left.\mathcal{N}_{\zeta}\right|_{X^{1,1}}$ is the restriction of $\mathcal{N}_{\zeta}$ - the symplectic quotient of $X$ by $\mathcal{G}$ - to $X^{1,1}$.

The above discussion motivates us to define a new $N_{c}=(2,0)$ model with the following action functional $S_{h}(\zeta, 0)$, modifying the original $N_{c}=(2,0)$ action functional $S$ in (3.78)

$$
\begin{aligned}
S_{h}(\zeta, 0)= & -i \boldsymbol{s}_{+} \overline{\boldsymbol{s}}_{+}\left\langle h_{\alpha \bar{\beta}} \chi_{-}^{\alpha}, \chi_{-}^{\bar{\alpha}}\right\rangle+i \boldsymbol{s}_{+}\left\langle\chi_{-}^{\alpha}, \mathfrak{S}_{\alpha}\right\rangle+i \overline{\boldsymbol{s}}_{+}\left\langle\chi_{-}^{\bar{\alpha}}, \mathfrak{S}_{\bar{\alpha}}\right\rangle \\
& -i\left\langle\phi_{++}, \mu-\zeta\right\rangle-i g_{i \bar{j}} \psi_{+}^{i} \psi_{+}^{\bar{i}},
\end{aligned}
$$

where we removed the $N_{c}=(2,0)$ gauge multiplet $\left(\phi_{--}, \eta_{-}, \bar{\eta}_{-}, D\right)$ and added the action functional $S(\zeta, 0)$ of the equivariant toy model. According to the previous discussion we see that the partition function defined by the new action $S_{h}(\zeta, 0)$ is equivalent to the expectation value of $\exp \left(\widehat{\varpi}_{\mathcal{G}}\right)$, evaluated by the original $N_{c}=(2,0)$ action functional $S$ (3.78).

Now we define more general action functional $S_{h}(\zeta, \varepsilon)$ by

$$
\begin{aligned}
S_{h}(\zeta, \varepsilon):= & -\boldsymbol{s}_{+} \overline{\boldsymbol{s}}_{+}\left\langle h_{\alpha \bar{\beta}} \chi_{-}^{\alpha}, \chi_{-}^{\bar{\alpha}}\right\rangle+i \boldsymbol{s}_{+}\left\langle\chi_{-}^{\alpha}, \mathfrak{S}_{\alpha}\right\rangle+i \overline{\boldsymbol{s}}_{+}\left\langle\chi_{-}^{\bar{\alpha}}, \mathfrak{S}_{\bar{\alpha}}\right\rangle \\
& -i\left\langle\phi_{++}, \mu-\zeta\right\rangle-i g_{\bar{i} \bar{j}} \psi_{+}^{i} \psi_{+}^{\bar{i}}+\frac{\varepsilon}{2}\left\langle\phi_{++}, \phi_{++}\right\rangle .
\end{aligned}
$$

We call the $N_{c}=(2,0)$ model with the above action functional $S_{h}(\zeta, \varepsilon)$ a holomorphic $N_{c}=(2,0)$ model, see [43] for the first example. Now we immediately see that the path integral of the holomorphic $N_{c}=$ $(2,0)$ model is governed by Witten's non-Abelian localization principle [35]. The first line of the above action functional localizes the path integral to $X^{1,1}$. Then, following the discussions in Sect. 2.2.3, the path integral can be written as the sum of contributions of the critical points $I=\langle\mu-\zeta, \mu-\zeta\rangle$ in $X^{1,1}$. Also from the discussions in Sect. 2.2.3 the $\varepsilon$-dependent term regularizes the path integral when $\mathcal{M}_{\zeta}$ develops singularities. 


\subsubsection{The Mapping Between the Two Models}

Now we will give more wider viewpoints which contain the original and holomorphic $N_{c}=(2,0)$ models as two special limits, following the original method of Witten [35]. Witten considered the case without the Fermi multiplets but for general manifolds. The Fermi multiplets will be purely spectators, and the specialization to a Kähler case will simplify the procedure.

Consider the following one-parameter family of $N_{c}=(2,0)$ supersymmetric action functional $S(\zeta)_{\lambda}$,

$$
\begin{aligned}
S(\zeta)_{\lambda}:= & S(\zeta)+\frac{\lambda}{2} \boldsymbol{s}_{+} \overline{\boldsymbol{s}}_{+}\left\langle\phi_{--}, \phi_{--}\right\rangle \\
= & -\boldsymbol{s}_{+} \overline{\boldsymbol{s}}_{+}\left\langle h_{\alpha \bar{\beta}} \chi_{-}^{\alpha}, \chi_{-}^{\bar{\alpha}}\right\rangle+i \boldsymbol{s}_{+}\left\langle\chi_{-}^{\alpha}, \mathfrak{S}_{\alpha}\right\rangle+i \overline{\boldsymbol{s}}_{+}\left\langle\chi_{-}^{\bar{\alpha}}, \mathfrak{S}_{\bar{\alpha}}\right\rangle \\
& -\boldsymbol{s}_{+} \overline{\boldsymbol{s}}_{+}\left(\left\langle\phi_{--}, \mu-\zeta-\frac{\lambda}{2} \phi_{--}\right\rangle-\left\langle\eta_{-}, \bar{\eta}_{-}\right\rangle\right) .
\end{aligned}
$$

If we set $\lambda=0$ we have the original $N_{c}=(2,0)$ model. For $\lambda \neq 0$ we can integrate out the $N_{c}=(2,0)$ gauge multiplet, and we are left with

$$
\begin{aligned}
S^{\prime}(\zeta)_{\lambda}= & -s_{+} \overline{\boldsymbol{s}}_{+}\left\langle h_{\alpha \bar{\beta}} \chi_{-}^{\alpha}, \chi_{-}^{\bar{\alpha}}\right\rangle+i \boldsymbol{s}_{+}\left\langle\chi_{-}^{\alpha}, \mathfrak{S}_{\alpha}\right\rangle+i \overline{\boldsymbol{s}}_{+}\left\langle\chi_{-}^{\bar{\alpha}}, \mathfrak{S}_{\bar{\alpha}}\right\rangle \\
& +\frac{1}{2 \lambda} \boldsymbol{s}_{+} \overline{\boldsymbol{s}}_{+}\langle\mu-\zeta, \mu-\zeta\rangle+\mathcal{O}\left(1 / \lambda^{2}\right)
\end{aligned}
$$

Since the additional $\lambda$-dependent term is closed by $s_{+}$and $\overline{\boldsymbol{s}}_{+}$, the path integral does not depend on $\lambda$ as long as $\lambda \neq 0$. The models with $\lambda=0$ and $\lambda \neq 0$ can be different since new fixed points can flow from the infinity $\lambda \rightarrow \infty$ in the field space [35].

If we take the limit $\lambda \rightarrow 0$, while $\lambda \neq 0$, we see that the dominant contributions to the path integral come from the critical points of $I=$ $\langle\mu-\zeta, \mu-\zeta\rangle$. Now we add $s_{+}$and $\bar{s}_{+}$-closed observables, $-\widehat{\varpi}+$ $\frac{\varepsilon}{2}\left\langle\phi_{++}, \phi_{++}\right\rangle$, to the above action functional,

$$
\begin{aligned}
S^{\prime}(\zeta, \varepsilon)_{\lambda}= & -\boldsymbol{s}_{+} \overline{\boldsymbol{s}}_{+}\left\langle h_{\alpha \bar{\beta}} \chi_{-}^{\alpha}, \chi_{-}^{\bar{\alpha}}\right\rangle+i \boldsymbol{s}_{+}\left\langle\chi_{-}^{\alpha}, \mathfrak{S}_{\alpha}\right\rangle+i \overline{\boldsymbol{s}}_{+}\left\langle\chi_{-}^{\bar{\alpha}}, \mathfrak{S}_{\bar{\alpha}}\right\rangle \\
& -i\left\langle\phi_{++}, \mu-\zeta\right\rangle-i\left\langle g_{i \bar{j}} \psi_{+}^{i}, \psi_{+}^{\bar{i}}\right\rangle+\frac{\varepsilon}{2}\left\langle\phi_{++}, \phi_{++}\right\rangle \\
& +\frac{1}{2 \lambda} \boldsymbol{s}_{+} \overline{\boldsymbol{s}}_{+}\langle\mu-\zeta, \mu-\zeta\rangle+\mathcal{O}\left(1 / \lambda^{2}\right)
\end{aligned}
$$

In the above the path integral should be independent of $\lambda \neq 0$. Consequently we see that the partition function of the above action functional 
can still be written as a sum of contributions from the critical points of $I$. Finally we may take the limit $\lambda \rightarrow \infty$ to remove all the $\lambda$-dependent terms and obtain the action functional $S_{h}(\zeta, \varepsilon)(4.3)$ of the holomorphic $N_{c}=(2,0)$ model. Thus we showed that the partition function of the holomorphic $N_{c}=(2,0)$ model can be written as a sum of contributions from the critical points of $I=\langle\mu-\zeta, \mu-\zeta\rangle$.

\subsection{Flows from $N_{c}=(2,2)$ to $N_{c}=(2,0)$ Models}

Consider an equivariant $N_{c}=(2,0)$ model as described in Sect. 2.4. with $\mathfrak{J}^{\alpha}=0$. Such a model was classified by a $\mathcal{G}$-equivariant Hermitian holomorphic bundle $E \rightarrow X$ with holomorphic section $\mathfrak{S}_{A}$. In a generic situation the model is equivalent to a non-linear $N_{c}=(2,0)$ model which target space $\mathcal{M}_{\zeta}$ is $\mathcal{M}_{\zeta}=\left(X \cap \mathfrak{S}^{-1}(0) \cap \mu^{-1}(\zeta)\right) / \mathcal{G}$. In this section we define a canonical embedding of such a model to $N_{c}=(2,2)$ model based on the tangent space $T E$ of the total space of bundle $E \rightarrow X$. Then we study the mapping from the $N_{c}=(2,2)$ to the $N_{c}=(2,0)$ model. We will see that the above circle of ideas leads us to find a $N_{c}=(2,0)$ model which is "equivalent" to the original $N_{c}=(2,0)$ model. From the viewpoint of the original $N_{c}=(2,0)$ model there is no a priori reason of such a "equivalence" to a completely different model. For simplicity we restricted to the linear model.

\subsubsection{Embedding of a $N_{c}=(2,0)$ Model to $N_{c}=(2,2)$ Model.}

The basic idea behind extension to $\mathcal{N}_{c}=(2,2)$ model is that one can regard the total space of holomorphic bundle $E \rightarrow X$ as the target space of a $\mathcal{N}_{c}=(2,2)$ model. Then we have to supply local holomorphic coordinates fields for fiber space of the bundle $E \rightarrow X$. Thus we introduce adjoint-valued bosonic spectral fields $B^{\alpha}$ and its superpartner $\chi_{+}^{\alpha}$. Now the former equivariant holomorphic section $\mathfrak{S}^{\alpha}\left(X^{i}\right)$ of bundle $E \rightarrow X$ corresponds to holomorphic vector on the target space $E$ but being supported only on $X$. Thus the $\mathcal{G}$-equivariant holomorphic vector $\mathfrak{S}^{\alpha}\left(X^{i}\right)$ should be extended over the whole space $E$. Furthermore $\mathcal{N}_{c}=$ $(2,2)$ supersymmetry requires that such holomorphic vector should be gradient vector of a non-degenerated $\mathcal{G}$-invariant holomorphic function $\mathcal{W}$, i.e, $\bar{s}_{+} \mathcal{W}=0$, of the target space $\mathbb{E}$. 
Now demanding $N_{c}=(2,2)$ supersymmetry will take care of everything. Recall that the $N_{c}=(2,0)$ model has a $\operatorname{Lie}(\mathcal{G})$-valued gauge multiplet associated with the group action of $\mathcal{G}$. We add a $\operatorname{Lie}(\mathcal{G})$ valued holomorphic multiplet $\bar{\sigma} \stackrel{s_{+}}{\longrightarrow} \eta_{+}$, to form a $N_{c}=(2,2)$ gauge multiplet

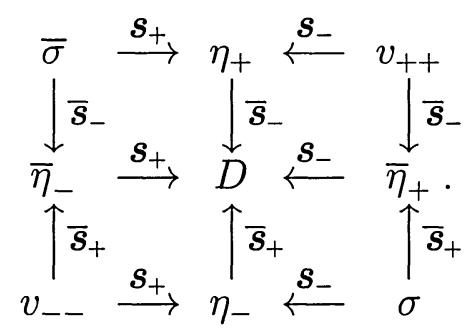

We had holomorphic multiplets $\left(X^{i} \stackrel{\boldsymbol{s}_{+}}{\longrightarrow} \psi_{+}^{i}\right), i=1, \ldots, n$, associated with the base space $X$ of $\mathbb{E} \rightarrow X$. By adding new Fermi multiplets $\left(\psi_{-}^{i} \stackrel{\boldsymbol{s}_{+}}{\longrightarrow} H^{i}\right)$, we extend them to $N_{c}=(2,2)$ chiral multiplets;

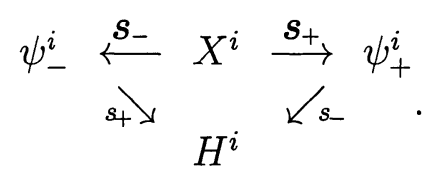

We also had Fermi multiplets $\left(\chi_{-}^{\alpha} \stackrel{s_{+}}{\longrightarrow} H^{\alpha}\right), \alpha=1, \ldots, r$, associated with the fibre of $\mathbb{E} \rightarrow X$. By adding new holomorphic multiplets $\left(B^{\alpha} \stackrel{s_{+}}{\longrightarrow} \chi_{+}^{\alpha}\right)$, we extend them to $N_{c}=(2,2)$ chiral multiplets;

$$
\chi_{-}^{\alpha} \underset{s_{+} \searrow}{\stackrel{s_{-}}{\longleftarrow}} B^{\alpha} \stackrel{\boldsymbol{s}_{+}}{\stackrel{s_{-}}{\longrightarrow}} \chi_{+}^{\alpha} .
$$

Now we consider the following $N_{c}=(2,2)$ supersymmetric action functional

$$
\begin{aligned}
S= & s_{+} \overline{\boldsymbol{s}}_{+} \boldsymbol{s}_{-} \overline{\boldsymbol{s}}_{-}\left(\sum_{i=1}^{n}\left\langle X^{i}, X^{\bar{i}}\right\rangle+\sum_{\alpha=1}^{r}\left\langle B^{\alpha}, B^{\bar{\alpha}}\right\rangle-\langle\sigma, \bar{\sigma}\rangle\right) \\
& +\boldsymbol{s}_{+} \boldsymbol{s}_{-} \mathcal{W}\left(X^{i}, B^{\alpha}\right)+\overline{\boldsymbol{s}}_{+} \overline{\boldsymbol{s}}_{-} \overline{\mathcal{W}}\left(X^{\bar{i}}, B^{\bar{\alpha}}\right) \\
& +\boldsymbol{s}_{+} \overline{\boldsymbol{s}}_{-}\langle t, \bar{\sigma}\rangle+\overline{\boldsymbol{s}}_{+} \boldsymbol{s}_{-}\langle\sigma, \bar{t}\rangle .
\end{aligned}
$$

To relate the above model with the initial $N_{c}=(2,0)$ model we assume the following conditions

$$
\frac{\partial \mathcal{W}}{\partial B^{\alpha}}=\mathfrak{S}_{\alpha}\left(X^{i}\right)
$$


where $\mathfrak{S}_{\alpha}$ is the holomorphic section of $\mathbb{E}$. This condition implies that $\mathcal{W}\left(X^{i}, B^{\alpha}\right)$ is linear in $B^{\alpha}$. We will utilize this property later. It is useful to rewrite the action functional $S$ (4.10) such that only the $N_{c}=(2,0)$ symmetry is manifest

$$
\begin{aligned}
S= & -i s_{+} \bar{s}_{+}\left(\left\langle\phi_{--}, \dot{\mu}_{X}+\mu_{F}-\zeta\right\rangle+\sum\left\langle\psi_{-}^{i}, \psi_{-}^{\bar{i}}\right\rangle\right. \\
& \left.+\sum\left\langle\chi_{-}^{\alpha}, \chi_{-}^{\bar{\alpha}}\right\rangle-\left\langle\eta_{-}, \bar{\eta}_{-}\right\rangle\right) \\
& +i s_{+}\left(\left\langle\psi_{-}^{i}, G_{i}\right\rangle+\left\langle\chi_{-}^{\alpha}, \mathfrak{S}_{\alpha}\right\rangle\right)+i \overline{\boldsymbol{s}}_{+}\left(\left\langle\psi_{-}^{\bar{i}}, G_{\bar{i}}\right\rangle+\left\langle\chi_{-}^{\bar{\alpha}}, \mathfrak{S}_{\bar{\alpha}}\right\rangle\right)
\end{aligned}
$$

where $\mu_{X}$ and $\mu_{F}$ are the momentum maps on $X$ and the fibre of $\mathbb{E}$, respectively, while

$$
G_{i}\left(X^{j}, B^{\alpha}\right):=\frac{\partial}{\partial X^{i}} \mathcal{W}\left(X^{j}, B^{\alpha}\right) .
$$

Note that $G_{i}$ is linear in $B^{\alpha}$ since $\mathcal{W}$ is linear in $B^{\alpha}$.

Applying the fixed point theorem we see that the path integral is localized to the solution space of the following equations, modulo the group action of $\mathcal{G}$

$$
\begin{aligned}
\mathfrak{S}_{\alpha}\left(X^{i}\right) & =0, \\
G_{i}\left(X^{j}, B^{\alpha}\right) & =0, \\
\mu_{X}\left(X^{i}, X^{\bar{i}}\right)+\mu_{F}\left(B^{\alpha}, B^{\bar{\alpha}}\right)-\zeta & =0,
\end{aligned}
$$

and

$$
\begin{aligned}
\varphi_{m}^{a} \mathcal{L}_{a}\left(X^{i}\right) & =0 \\
\varphi_{m}^{a} \mathcal{L}_{a}\left(B^{\alpha}\right) & =0 \\
{\left[\varphi_{m}, \varphi_{n}\right] } & =0 \\
{\left[\varphi_{m}, \bar{\varphi}_{m}\right] } & =0 .
\end{aligned}
$$

This model, for a generic value of $\zeta$ implying $\varphi_{m}=0$ as usual, reduce to the non-linear $N_{c}=(2,2)$ model whose target space $\mathfrak{M}_{\zeta}$ is the space of all solutions of the equations (4.14) modulo $\mathcal{G}$-symmetry. 


\subsubsection{Perturbation to a $N_{c}=(2,0)$ Model}

Now we want to perturb the $N_{c}=(2,2)$ model above to a $N_{c}=(2,0)$ model by breaking the $N_{c}=(0,2)$ supersymmetry generated by $s_{-}$and $\bar{s}_{-}$. This can be done by giving bare "mass" to all the newly introduced multiplets given by

$$
\left(\bar{\sigma}, \eta_{+}\right), \quad\left(\psi_{-}^{i}, H^{i}\right), \quad\left(B^{\alpha}, \chi_{+}^{\alpha}\right)
$$

and their conjugates. Then the model flows to the original $N_{c}=(2,0)$ model if we take the bare "mass" to infinity. Such bare mass terms will have special geometrical meaning.

Note that there is a natural $U(1)=S^{1}$ group acting on $B^{\alpha}$, while leaving fixed the $X^{i}$, such that the momentum map $\mu_{F}$ remains invariant. This $S^{1}$-action is given by

$$
S^{1}:\left(X^{i}, B^{\alpha}\right) \rightarrow\left(X^{i}, \xi B^{\alpha}\right)
$$

where $\xi \bar{\xi}=1$. Note that the above $S^{1}$-action does not change the first and the last equations of (4.14). The LHS of the second equation of (4.14) will be multiplied by $\zeta$, which does not alter the solution space of the equation. Thus the $S^{1}$-action is a symmetry of the effective target space $\mathfrak{M}_{\zeta}$.

It is important to note that the above $U(1)$ needs not be a symmetry of our $N_{w s}=(2,2)$ model. To be such a symmetry, the $S^{1}$ action (4.17) should be extended to all the superpartners. That is, $\psi_{ \pm}^{i}$ and $H^{i}$ should be invariant under $U(1)$ while $\chi_{ \pm}^{\alpha}$ and $H^{\alpha}$ should carry the $U(1)$-charge 1 . We, however, demand that the above $U(1)$ is compatible with the $N_{c}=(2,0)$ supersymmetry generated by $\boldsymbol{s}_{+}$and $\bar{s}_{+}$supercharges. From the expression (4.12) of $S$ with manifest $N_{c}=(2,0)$ symmetry we see that the $\psi_{-}^{i}$ should carry $U(1)$ charge -1 , since $G_{i}\left(X^{j}, B^{\alpha}\right)$ is linear in $B^{\alpha}$. Then, by examining the supersymmetry transformation laws for the supercharges $\boldsymbol{s}_{+}$and $\overline{\boldsymbol{s}}_{+}$, we see that the $S^{1}$-symmetry (4.17) should be extended to all the $N_{c}=(2,0)$ multiplets in (4.16) as follows

$$
\begin{aligned}
& S^{1}:\left(B^{\alpha}, \chi_{+}^{\alpha}\right) \rightarrow \xi\left(B^{\alpha}, \chi_{+}^{\alpha}\right) \\
& S^{1}:\left(\psi_{-}^{i}, H^{i}\right) \rightarrow \bar{\xi}\left(\psi_{-}^{i}, H^{i}\right), \\
& S^{1}:\left(\bar{\sigma}, \eta_{+}\right) \rightarrow \bar{\xi}\left(\bar{\sigma}, \eta_{+}\right) .
\end{aligned}
$$


That is, we give $U(1)$-charges to the fields in (4.16) while all the other fields remain neutral. Clearly this can't be done while maintaining the full $N_{c}=(2,2)$ supersymmetry.

Recall that the $N_{c}=(2,0)$ supercharges $\boldsymbol{s}_{+}$and $\overline{\boldsymbol{s}}_{+}$satisfy now familiar anti-commutation relations

$$
s_{+}^{2}=0, \quad\left\{s_{+}, \bar{s}_{+}\right\}=-i \phi_{++}^{a} \mathcal{L}_{a}, \quad \bar{s}_{+}^{2}=0,
$$

defining the $\mathcal{G}$-equivariant Dolbeault cohomology. Since we have an additional $S^{1}$ acting on our system it is natural to extend the above to $\mathcal{G} \times S^{1}$-equivariant cohomology. Then the new supercharges, still to be denoted $s_{+}$and $\bar{s}_{+}$, satisfy the following anti-commutation relations

$$
s_{+}^{2}=0, \quad\left\{s_{+}, \bar{s}_{+}\right\}=-i \phi_{++}^{a} \mathcal{L}_{a}-i m \mathcal{L}_{S^{1}}, \quad \bar{s}_{+}^{2}=0,
$$

where we introduced a parameter $m$ taking values in $\operatorname{Lie}\left(S^{1}\right)$. The supersymmetry transformation laws should be modified accordingly.

Finally we define the following $N_{c}=(2,0)$ supersymmetric action functional

$$
S(m, \bar{m})=S^{\prime}+\bar{m} \boldsymbol{s}_{+} \overline{\boldsymbol{s}}_{+}\left(\sum_{\alpha=1}^{r}\left\langle B^{\alpha}, B^{\bar{\alpha}}\right\rangle-\langle\sigma, \bar{\sigma}\rangle\right),
$$

where $S^{\prime}$ is defined by the same formula as the action functional in (4.12) but with the modified supersymmetry. The new action functional $S(m, \bar{m})$, compared to the $N_{c}=(2,2)$ symmetric action $S$, is

$$
\begin{aligned}
S(m, \bar{m})= & S+m \bar{m} \sum_{\alpha}\left\langle B^{\alpha}, B^{\bar{\alpha}}\right\rangle-i \bar{m} \sum_{\alpha}\left\langle\chi_{+}^{\alpha}, \chi_{+}^{\bar{\alpha}}\right\rangle+m \bar{m}\langle\sigma, \bar{\sigma}\rangle \\
& -i \bar{m}\left\langle\bar{\eta}_{+}, \eta_{+}\right\rangle-i m\left\langle\phi_{--}, \mu_{F}-[\sigma, \bar{\sigma}]\right\rangle \\
& +i \bar{m}\left\langle\phi_{++}, \mu_{F}-[\sigma, \bar{\sigma}]\right\rangle+i m \sum_{i}\left\langle\psi_{-}^{i}, \psi_{-}^{\bar{i}}\right\rangle
\end{aligned}
$$

containing the desired mass terms. We note that the mass terms contain the Hamiltonian $H_{S_{1}}$ of the $S^{1}$ symmetry on the space of all $B^{\alpha}$ and $\sigma$;

$$
H_{S^{1}}=i \sum_{\alpha}\left\langle B^{\alpha}, B^{\bar{\alpha}}\right\rangle+i\langle\sigma, \bar{\sigma}\rangle .
$$

This fact will play a crucial role later. 
Now we examine the equation for fixed points. Since we only have $\boldsymbol{s}_{+}$and $\overline{\boldsymbol{s}}_{+}$supersymmetry the path integral is localized to the fixed point locus of those symmetries, modulo the $\mathcal{G}$ symmetry. We have

$$
\begin{aligned}
\bar{\sigma}^{a} \mathcal{L}_{a}\left(X^{i}\right) & =0, \\
\bar{\sigma}^{a} \mathcal{L}_{a}\left(B^{\alpha}\right) & =0, \\
\mathfrak{S}_{\alpha}\left(X^{i}\right) & =0, \\
G_{i}\left(X^{j}, B^{\alpha}\right) & =0, \\
\mu_{X}\left(X^{i}, X^{\bar{i}}\right)+\mu_{F}\left(B^{\alpha}, B^{\bar{\alpha}}\right)-[\sigma, \bar{\sigma}]-\zeta & =0,
\end{aligned}
$$

and

$$
\begin{aligned}
{[\phi, \bar{\phi}] } & =0, \\
\phi^{a} \mathcal{L}_{a}\left(X^{i}\right) & =0 \\
\phi^{a} \mathcal{L}_{a}\left(B^{\alpha}\right)+m B^{\alpha} & =0 \\
{[\phi, \bar{\sigma}]-m \sigma } & =0 .
\end{aligned}
$$

The set of equations in (4.24) cut out a subspace of the space of all $X^{i}, B^{\alpha}$ and $\sigma$. After modding out the $\mathcal{G}$-symmetry we get the effective target space $\widetilde{\mathfrak{M}}_{\zeta}$ of our $N_{c}=(2,0)$ model. Following the previous general discussions we expect that $\widetilde{\mathfrak{M}}_{\zeta}$ is a Kähler manifold at least for the generic case. The set of equations in (4.25) represent gauge degrees of freedom. In particular those equations implies the path integral is localized to the fixed point of $S^{1}$ action on $\widetilde{\mathfrak{M}}_{\zeta}$.

We always have trivial fixed points, namely $B^{\alpha}=\sigma=0$. We call such fixed points branch (i). In branch (i) the path integral is localized to the solution space of the following equations, modulo $\mathcal{G}$-symmetry,

$$
\begin{aligned}
\phi^{a} \mathcal{L}_{a}\left(X^{i}\right) & =0, \\
\mu_{X}\left(X^{i}, X^{\bar{i}}\right)-\zeta & =0 .
\end{aligned}
$$

which are exactly the generic fixed point equations for the original $N_{c}=(2,0)$ model. There are other fixed points with $B^{\alpha}, \bar{\sigma} \neq 0$ when the $S^{1}$-action can be undone by the $\mathcal{G}$ action. The last two equations in (4.25) exactly stand for such property. We call such fixed points branch (ii).

The above localization principle can also be obtained from a different viewpoint. We consider a limit $|m| \rightarrow \infty$. Then the dominant 
contributions to the path integral come from the set of critical points of the Hamiltonian $H_{S^{1}}$ defined by (4.23). It is well-known that the critical points of the Hamiltonian of a $S^{1}$ action are exactly the same as the fixed points of the $S^{1}$ action. One may evaluate the partition function in such a limit and set $|m|=0$ afterwards, to get the partition function of the $N_{c}=(2,2)$ model.

Now we assume that everything is generic, so that we do not have any zero-modes of anti-ghosts, $\chi_{-}^{\alpha}, \psi_{-}^{i}$, as well as any zero-modes of the $N_{c}=(2,0)$ gauge multiplets. Then the partition function of the action functional $S(m, \bar{m})$ in (4.25) reduces to the following integral

$$
Z=\int_{\widetilde{\mathfrak{M}}_{\zeta}} \exp \left(i m H_{S^{1}}+i \sum_{\alpha}\left\langle\chi_{+}^{\alpha}, \chi_{+}^{\bar{\alpha}}\right\rangle+i\left\langle\bar{\eta}_{+}, \eta_{+}\right\rangle\right),
$$

where we regard $m$ and $\bar{m}$ as independent numbers and scaled away the overall $\bar{m}$. The above resembles the $\mathrm{DH}$ integration formula on $\widetilde{\mathfrak{M}}_{\zeta}$. We see, however, that there is a missing term since the fermionic terms above correspond to the Kähler form only on the subspace of $\widetilde{\mathfrak{M}}_{\zeta}$ given by $X^{i}=0$. We can provide the missing term by evaluating the correlation function of $\exp \left(i\left\langle\phi_{++}, \mu_{X}\right\rangle+i \sum\left\langle\psi_{+}^{i}, \psi_{+}^{\bar{i}}\right\rangle\right)$, where the exponent is the $\mathcal{G}$-equivariant Kähler form $\widehat{\varpi}_{\mathcal{G}}^{X}$ on $X$. Note that it is an observable of the original $N_{c}=(2,0)$ model we started from. Assuming the same generic situation as above, the correlation function reduces to the following integral

$$
\left\langle e^{\widehat{\boldsymbol{\omega}}_{\mathcal{G}}^{X}}\right\rangle=\int_{\widetilde{\mathfrak{M}}_{\zeta}} \exp \left(i m H_{S^{1}}+\widetilde{\varpi}\right)
$$

where $\widetilde{\varpi}$ denote the Kähler form on $\widetilde{\mathfrak{M}}_{\zeta}$. Now we have exactly the DH integration formula [36]. The integral can be written as the sum of contributions from the fixed points of the $S^{1}$-action.

We saw that we have two branches. In branch (i) the fixed point locus is the effective target space $\mathcal{M}_{\zeta}$ of the original $N_{c}=(2,0)$ model. The Hamiltonian $H_{S_{1}}$ in this branch is simply zero. Thus we are evaluating the symplectic volume of $\mathcal{M}_{\zeta}$. This is a correlation function of the original $N_{c}=(2,0)$ model. In branch (ii) the value $H_{S^{1}}^{f}$ of $H_{S^{1}}$ at a fixed point is non-zero. So the integral for each fixed point is weighted by a phase factor $\exp \left(i m H_{S^{1}}^{f}\right)$. For both branches the integral is weighted by a one loop determinant coming from the transverse 
degrees of freedom. We note that such a determinant contains factors of $m$ with certain weights depending on the particular fixed points. After evaluating the $\mathrm{DH}$ integral we can set $m=0$. Then we may obtain many relations by imposing that the poles should be cancelled order by order between the two different branches, since the limit $m \rightarrow 0$ should be smooth in the path integral of the massive $N_{c}=(2,0)$ model. The partition function of the $N_{c}=(2,2)$ model is given by a sum of terms with order zero in $m$. One can also obtain the symplectic volume of $\mathcal{M}_{\zeta}$ in terms of a sum of contributions coming from branch (ii).

In the real situation life is more complicated since it is difficult to achieve the generic conditions and the space $\widetilde{\mathfrak{M}}_{\zeta}$ may be non-compact. Its is in principle possible to elaborate on the above procedure and perform the integral. Even if we can't do such an integral due to technicalities we can at least see that the essential information on the correlation function of the original $N_{c}=(2,0)$ model is contained in the fixed points which belongs to branch (ii).

\section{Acknowledgement}

This article is partly based on the introductory chapters of my $\mathrm{PhD}$ thesis and lectures given at KIAS. It is my great pleasure to thanks my adviser Herman Verlinde for various suggestions and encouragement. I am grateful to KIAS for hospitality and a financial support during my visit. I am grateful to Robbert Dijkgraaf, Christiaan Hofman, Seungjoon Hyun and Bernd Schroers for fruitful collaborations on the related subjects. I am also grateful to Christiaan Hofman for correcting English and an error after a patient proof-reading. This work is supported by a pionier fund of NWO and DOE grant \# DE-FG0292ER40699.

\section{References}

[1] E. Witten, Topological quantum field theory, Commun. Math. Phys., 117 (1988), 353. 
[2] E. Witten, Introduction to cohomological field theories, Int. J. Mod. Phys., A6 (1991), 2775.

[3] E. Witten, Mirror manifolds and topological field theory, hep-th/9112056.

[4] W. Lerche, C. Vafa, and N.P. Warner, Chiral rings in N=2 superconformal theories, Nucl. Phys., B324 (1989) 427.

[5] Essays on mirror manifolds, S.-T. Yau ed., International Press, Hong Kong, 1992.

[6] Mirror symmetry II, B. Greene and S.-T. Yau eds, AMS/IP Studies in Adv. Math. 1., AMS, Providence, RI; International Press, Cambridge, MA (1997).

[7] C. Vafa and E. Witten, A strong coupling test of S-Duality, Nucl. Phys., B431 (1994), 3-77, hep-th/9408074.

[8] S.K. Donaldson, Polynomial invariants for smooth 4-manifolds, Topology, 29 (1990), 257.

[9] P. Kronheimer and T. Mrowka, Recurrence relations and asymptotics for four manifold invariants, Bull. Am. Math. Soc., 30 (1994), 215.

[10] E. Witten, Supersymmetric Yang-Mills theory on a four-manifold, J. Math. Phys., 35 (1994), 5101, hep-th/9303195.

[11] N. Seiberg and E. Witten, Electric-magenectic duality, monopole condensation, and confinement in $N=2$ supersymmetric YangMills theory, Nucl. Phys., B 426 (1994), 19.

[12] E. Witten, Monoples and four manifolds, Math. Research Lett., 1 (1994), 769, hep-th/9411102.

[13] E. Witten, The $N$ matrix model and gauged WZW models, Nucl. Phys., B 371 (1992), 191.

[14] B. Zumino, Supersymmetry and Kähler Manifolds, Phys. Lett., B 87 (1979), 203.

[15] L. Alvarez-Gaume, D.Z. Freedman, Geometrical structure and ultraviolet finiteness in the supersymmetric sigma model, Comm. Math. Phys., 80 (1981), 443. 
[16] N.J. Hitchin, A. Karlhede, U. Lindström, and M. Rocek, HyperKähler metrics and supersymmetry, Comm. Math. Phys., 108 (1987), 535 .

[17] S.J. Gates, Jr, C.M. Hull, and M. Rocek, Twisted multiplets and new supersymmetric non-linear $\sigma$-models, Nucl. Phys., B 248 (1984), 157.

[18] E. Witten Phases of $N=2$ theories in two dimensions, Nucl. Phys., B403 (1993), 159-222, hep-th/9301042.

[19] E. Witten, The Verlinde algebra and the cohomology of the Grassmannian, hep-th/9312104.

[20] E. Witten, Supersymmetry and Morse theory, J. Differ. Geom., 17 (1982), 692.

[21] M. Atiyah and L. Jeffreys, Topological Lagrangians and cohomology, J. Geom. Phys., 7 (1990), 119.

[22] S. Cordes, G. Moore, S. Ramgoolam, Large $N 2 D$ Yang-Mills theory and topological string theory, hep-th/9402107; Lectures on 2D Yang-Mills theory, equivariant cohomology and topological field theories, hep-th/9411210.

[23] R. Dijkgraaf and G. Moore, Balanced topological field theories, Commun. Math. Phys., 185 (1997), 411-440, hep-th/9608169.

[24] V. Mathai and D. Quillen, Superconnections, Thom classes and equivariant differential forms, Topology, 25 (1986), 85.

[25] W. Fulton, Intersection Theory, Springer-Verlag, 1984.

[26] J.-L. Koszul, Crochet de Schouten-Nijenhuis et cohomologie, in 'Elie Cartan t les matheématique d'aujourd'huis', Asterisque, (1985), 251.

[27] E. Witten, Dynamical breaking of supersymmetry, Nucl. Phys., B 188 (1981), 513.

[28] E. Witten, Constraints on supersymmetry breaking, Nucl. Phys., B 202 (253), 1982. 
[29] S. Kobayashi, Differential geometry of complex vector bundles, Iwanami Shoten, Publishers and Princeton Univ. Press, 1987.

[30] M. Atiyah and R. Bott, The momentum map and equivariant cohomology, Topology 23 (1984) 1.

[31] N. Berline, E. Getzler and M. Vergne, Heat kernels and Dirac operators, Springer-Verlag, 1992.

[32] J.-S. Park, N=2 topological Yang-Mills theory on compact Kähler surfaces, Comm. Math. Phys. 163 (1994) 113, hep-th/9304060.

[33] S. Hyun and J.-S. Park, N=2 topological Yang-Mills theories and Donaldson's polynomials, J. Geom. Phys. 20 (1996) 31-53, hep-th/9404009.

[34] J. Marsden and A.D. Weinstein, Reductiion of symplectic manifolds with symmetry, Reports on Math. Physics 5 (1974) 121.

[35] E. Witten, Two dimensional gauge theories revisited, hep-th/9204084.

[36] J.J. Duistermaat and G.J. Heckman, On the variation in the cohomology of the symplectic form of the reduced phase space, Invent. Math. 69 (1982) 259; Addendum, Invent. Math. 72 (1983) 153.

[37] M. Atiyah, Convexity and commuting Hamiltonians, Bull. London Math. Soc. 14 (1982) 1.

[38] F. Kirwan, Cohomology of Quotients in Symplectic and Algebraic Geometry, Math. Notes 31. Princeton Univ. Press, (Princeton, 1984).

[39] R. Dijkgraaf, B.J. Schroers and J.-S. Park, N=4 supersymmetric Yang-Mills theory on a Kähler surface, hep-th/9801066.

[40] M. Blau and G. Thompson, $N=2$ topological gauge theory, the Euler characteristic of moduli spaces, and the Casson invariant, Commun. Math. Phys. 152 (1993) 41-72.

[41] D. Mumford and J. Fogarty, Geometrical Invariant Theory, (Springer, 1982).

[42] P. Newstead, Introduction to moduli problems and orbit spaces, (Tata Institute, 1978).

[43] J.-S. Park, Holomorphic Yang-Mills theory on compact Kähler manifolds, Nucl. Phys. B423 (1994) 559, hep-th/9305095. 